\title{
Symmetry Breaking, Coexisting Bubbles, Multistability, and Its Control for a Simple Jerk System with Hyperbolic Tangent Nonlinearity
}

\author{
Léandre Kamdjeu Kengne $\left(\mathbb{D},{ }^{1,2}\right.$ Jacques Kengne, ${ }^{1}$ Justin Roger Mboupda Pone, \\ and Hervé Thierry Kamdem Tagne $\mathbb{1}^{3}$ \\ ${ }^{1}$ Unité de Recherche d'Automatique et Informatique Appliquée (UR-AIA), Department of Electrical Engineering, \\ IUT-FV Bandjoun, University of Dschang, P.O. Box 134, Bandjoun, Cameroon \\ ${ }^{2}$ Unité de Recherche de Matière Condensée, d'Electronique et de Traitement du Signal (UR-MACETS), Department of Physics, \\ University of Dschang, P.O. Box 67, Dschang, Cameroon \\ ${ }^{3}$ Unité de Recherche de Mécanique et de Modélisation des Systèmes Physiques (UR-2MSP), Department of Physics, \\ University of Dschang, P.O. Box 67, Dschang, Cameroon
}

Correspondence should be addressed to Léandre Kamdjeu Kengne; lkamdjeu@gmail.com

Received 31 December 2019; Revised 20 February 2020; Accepted 26 February 2020; Published 23 April 2020

Academic Editor: Dimitri Volchenkov

Copyright (c) 2020 Léandre Kamdjeu Kengne et al. This is an open access article distributed under the Creative Commons Attribution License, which permits unrestricted use, distribution, and reproduction in any medium, provided the original work is properly cited.

\begin{abstract}
Symmetry is an important property found in a large number of nonlinear systems. The study of chaotic systems with symmetry is well documented. However, the literature is unfortunately very poor concerning the dynamics of such systems when their symmetry is altered or broken. In this paper, we investigate the dynamics of a simple jerk system with hyperbolic tangent nonlinearity (Kengne et al., Chaos Solitons, and Fractals, 2017) whose symmetry is broken by adding a constant term modeling an external excitation force. We demonstrate that the modified system experiences several unusual and striking nonlinear phenomena including coexisting bifurcation branches, hysteretic dynamics, coexisting asymmetric bubbles, critical transitions, and multiple (i.e., up to six) coexisting asymmetric attractors for some suitable ranges of system parameters. These features are highlighted by exploiting common nonlinear analysis tools such as graphs of largest Lyapunov exponent, bifurcation diagrams, phase portraits, and basins of attraction. The control of multistability is investigated by using the method of linear augmentation. We demonstrate that the multistable system can be converted to a monostable state by smoothly adjusting the coupling parameter. The theoretical results are confirmed by performing a series of PSpice simulations based on an electronic analogue of the system.
\end{abstract}

\section{Introduction}

Recently, a particular attention has been paid to the study of nonlinear and chaotic dynamic systems. This is due to the rapid development of increasingly powerful computers on the one hand and on the other hand to the many potential applications in several fields of science and engineering. These systems are capable of several forms of complexity such as chaos, hyperchaos, multirhythmicity, bifurcations, intermittency, hysteresis, and multistability [1-3]. Concerning the latter feature, it should be noted that a multistable dynamic system is capable of displaying two or more attractors for the same set of parameters. In this situation, each of the coexisting attractors is connected with an attraction basin that represents all the initial conditions leading to the underlined attractor [4]. Fixed points, limit cycles, toruses, and chaotic attractors can coexist in the same system, in various combinations depending on the choice of parameters. The term extreme multistability refers to the situation where an infinite number of attractors coexist [5-9]. Multistability is relevant from the view point of practical application as it may give rise to unexpected and 
even disastrous consequences [10]. If the phenomenon of multistability is encountered in dynamic systems with no symmetry property, it should be noted that symmetric systems are much more likely to develop this phenomenon [10]. Concerning the symmetry property, it should be mentioned that it is shared by many systems in several fields of science and engineering $[2,4]$. In addition to multistability mentioned above, symmetric dynamic systems exhibit interesting behaviors such as period doubling, spontaneous symmetry breaking, merging crisis, hysteresis, and intermittency [1]. The study of symmetrical systems is well documented. However, to the best of the authors' knowledge, the literature is very poor concerning the behavior of these systems when their symmetry is altered or broken. The symmetry break purposefully induced in a nonlinear dynamical system may be adjusted to discover many complex nonlinear phenomena (e.g., multirhythmicity, bursting, coexisting bubbles, hysteresis, critical transitions, and coexisting multiple asymmetric attractors) as previously discussed in several nonlinear systems [11-17].

In this work, we consider a simple jerk system with hyperbolic tangent nonlinearity [18] whose symmetry is broken by the introduction of an additive constant $k$. We address the chaos generation mechanism, the formation of bubbles of bifurcation, and the coexistence of multiple attractors in both the symmetric $(k=0)$ and the asymmetric $(k \neq 0)$ regimes of operation. For convenience, recall that jerk dynamic systems [19-23] refer to 3D ordinary differential equations (ODEs) in the form $\dddot{x}=J(x, \dot{x}, \ddot{x})$ where the nonlinear vector function $J(\cdot)$ indicates the "jerk" (i.e., the time derivative of the acceleration). The hyperbolic tangent function is relevant in numerous problems such as nonideal operational amplifiers, activation function in neural network, magnetization in ferromagnetic systems, and solar wind-driven magnetosphere-ionosphere systems [24-28]. Multistability in simple jerk dynamic systems has recently drained tremendous research interest in varied fields of science and technology resulting in several publications. On this line, Kengne and colleagues reported the coexistence of four self-excited mutually symmetric attractors in a jerk system possessing a cubic nonlinearity [23] based on both numerical and experimental methods. This striking feature of multiple attractors is mainly due to the system's symmetry and thus is also obtained with a hyperbolic sine [29], a hyperbolic tangent [18], a composite tanh-cubic nonlinearity [21], or a voltage controlled memristor [30], whose intrinsic current-voltage characteristics has the form of a pinched hysteresis loop. Despite the pertinence and the importance of the abovementioned results, we would like to stress that all cases of multistability discussed so far is restricted to symmetric jerk systems; also, multistability in jerk dynamic systems in case of a broken symmetry is very little studied. Motivated by previous results on jerk dynamical systems, this paper focuses on the effects engendered by symmetry break in a simple autonomous jerk system with hyperbolic tangent nonlinearity previously analyzed in [18]. Thus, the novel chaotic flow is smoothly tuned to behave either symmetrically or to develop no symmetry property using a single parameter. Importantly, the investigations clearly reveal that the modified system can experience coexisting bubbles of bifurcation, coexisting multiple (symmetric or asymmetric) attractors, and crises phenomena not found in the original symmetric system [18]. Despite the fact that the addition of a constant term may be viewed as a purely mathematical technique to induce new nonlinear phenomena, one of the key motivations is that symmetries are rarely exact in real physical systems, and some symmetrybreaking imperfections are always present [31-33].

The structure of the paper is as follows. Section 2 describes the evolution equations of the modified jerk system with hyperbolic tangent and analyses possible symmetries. Analytical conditions for the occurrence of Hopf-type bifurcations are established, and the stability of the equilibrium points is investigated with respect to parameters. In Section 3, numerical results concerning the bifurcation behaviour of the model, the coexistence of numerous attractors, and the coexistence of bubbles of bifurcation are presented. The control of multistability based on the linear augmentation scheme is described in Section 4. Section 5 is concerned with the experimental study of the modified system. A convenient electronic circuit (i.e., the analogue simulator) is designed for investigating the extremely complex dynamics of the system. PSpice simulation experiment supports the results of the theoretical study. Finally, Section 6 presents the conclusions of the whole work.

\section{Description and Analysis of the Model}

2.1. The Model. The state equation of the autonomous jerk system, which is considered in this work, is expressed by the following third-order nonlinear system (ODEs) with a single nonlinear function:

$$
\begin{gathered}
\left\{\begin{array}{l}
\dot{x}_{1}=x_{2}, \\
\dot{x}_{2}=a x_{3}, \\
\dot{x}_{3}=-\gamma x_{2}-\mu x_{3}+\varphi_{k}\left(x_{1}\right),
\end{array}\right. \\
\varphi_{k}\left(x_{1}\right)=-k-3\left(x_{1}-2 \tanh \left(x_{1}\right)\right),
\end{gathered}
$$

where $a, \mu$, and $\gamma$ denote (real) positive control parameters. Notice that the nonlinearity is smooth and involves only one state variable (i.e., $x_{1}$ ). Here, $k$ is the symmetry control parameter of the model. Specifically, for $k=0$, system (1) exhibits a perfect symmetry and reduces to the case previously studied by Kengne and coworkers [18]. The case $k \neq 0$ corresponds to an asymmetric model for which more complex nonlinear phenomena arise (that cannot be explained by using the symmetry arguments) including, for instance, the presence of multiple coexisting asymmetric attractors, coexisting bifurcation branches, and crisis events (see Section 4). The graphical representations of the nonlinear function $\varphi_{k}(\cdot)$ are provided in Figure 1 for several discrete values of parameterk. Interestingly, we would like to quote that the hyperbolic tangent nonlinearity has also been considered in many problems related to neural networks and Chua's system as well [24-28]. The inclusion of this nonlinear term in model (1) engenders the extremely complex 


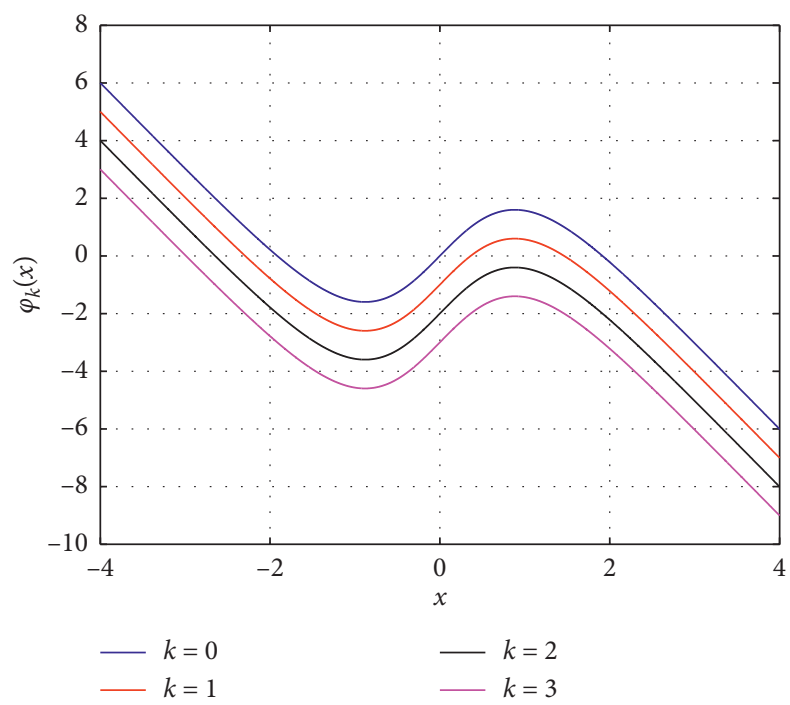

Figure 1: Graphical representation of the nonlinear function $\varphi_{k}(x)=-k-3 x+6 \tanh (x)$ for four discrete values of the symmetry parameter $k$. Notice that the number (one, two, or three) of zeros of $\varphi_{k}(\cdot)$ depends crucially on the value of parameter $k$.

and striking bifurcation patterns developed by the whole system. The most "elegant" form [4] of system (1) is achieved by expressing it as a jerk equation:

$$
\dddot{x}=-a \gamma \dot{x}-\mu \ddot{x}+a \varphi_{k}(x) \text {. }
$$

Finally, it should be remarked that the state variable $x_{1}$ appears solely in the third equation and, consequently, represents an offset boosted variable [34, 35].

2.2. Symmetry and Dissipation. As previously indicated above, it can easily be seen that systems (1a) and (1b) are symmetric with respect to the origin of the coordinates for the special case $k=0.0$. For this singular case, systems (1a) and (1b) represent an inversion invariant nonlinear dynamic system, provided that it remains unchanged when performing the coordinate substitution: $\left(x_{1}(t), x_{2}(t), x_{3}(t)\right) \Longleftrightarrow$ $\left(-x_{1}(t),-x_{2}(t),-x_{3}(t)\right)$. As a result, all attractors of systems (1a) and (1b) occur either as individual symmetric attractors or as mutually symmetric couples. This property is the key ingredient to justify the presence of multiple coexisting stable states appearing for some suitable sets of system parameters. More importantly, a suitable exploitation of the symmetry property of the evolution equation simplifies considerably the numerical experiment (e.g., the stability analysis, the calculation of phase space trajectory, and the basins of attraction as well).

More generally, systems (1a) and (1b) are nonsymmetric for any value of $k \neq 0.0$. However, we notice the invariance of systems (1a) and (1b) following the coordinate transformation $\left(x_{1}(t), x_{2}(t), x_{3}(t), k\right) \Longleftrightarrow\left(-x_{1}(t),-x_{2}(t),-\right.$ $\left.x_{3}(t),-k\right)$. Consequently, we restrict our analysis for positive values of parameter $k$ all over this work. The dynamics for negative values of $k$ can be deduced from the latter transformation.

The divergence of the vector field (1a) and (1b) is computed as follows:

$$
\begin{aligned}
\Lambda & =\frac{\partial \dot{x}_{1}}{\partial x_{1}}+\frac{\partial \dot{x}_{2}}{\partial x_{2}}+\frac{\partial \dot{x}_{3}}{\partial x_{3}}, \\
& =\frac{\partial\left(x_{2}\right)}{\partial x_{1}}+\frac{\partial\left(a x_{3}\right)}{\partial x_{2}}+\frac{\partial\left(-k-\gamma x_{2}-\mu x_{3}-3 x_{1}+6 \tanh \left(x_{1}\right)\right)}{\partial x_{3}}, \\
& =-\mu<0 .
\end{aligned}
$$

It follows that, for any point $x=\left(x_{1}, x_{2}, x_{3}\right)$ in phase space, the divergence is always negative. Accordingly, system (1) is dissipative and consequently can develop attractors [1-3].

2.3. Fixed Point Analysis. The study of equibria always represents the first issue to be addressed when performing the investigation of a nonlinear dynamic system. Their study yields preliminary insights into the dynamics of complete system [1-4]. By equating to zero all the derivatives in system (5), we found that (see Figure 1), for $|k|<k_{\max } \approx 7.57$, there exist three different rest points $E_{n}=\left(x_{n}, 0,0\right)(n=0,1,2)$, where $x_{j}$ verifies the following transcendental equation:

$$
k+3(x-2 \tanh (x))=0 .
$$

In view of the graph presented in Figure 1, we notice that, for $|k|=k_{\max }$, the system has two fixed points, while a single equilibrium point exists in case $|k|>k_{\max }$. Throughout the rest of this work, we restrict our analysis for values of $k$ where the system exhibits three fixed points. It should be remarked that, for $k=0.0$, the system displays three symmetric fixed points amongst which the origin [18]. Using the set of parameters defined above, the roots equation (4) have been numerically obtained for two discrete values of $k$ (i.e., $k=0.00$ and $k=0.25$ ) by using the "fzero" build in function of Matlab. Recall that the "fzero" function is a MATLAB subroutine to search for the zeros of a single variable real-value function. As 
sample results, we have obtained the following fixed points: (i) $E_{0}(0,0,0)$ and $E_{1,2}( \pm 1.915,0,0)$ for $k=0.0$; (ii) $E_{0}(0.083,0,0), E_{1}(1.813,0,0)$, and $E_{2}(-2.013,0,0)$ for $k=0.25$. We notice that the positions of the equilibrium points in state space are defined solely by the value of parameter $k$. Evaluated at any given fixed point $E(\bar{x}, 0,0)$, the Jacobian matrix of system (1) takes the following form:

$$
M_{J}=\left[\begin{array}{ccc}
0 & 1 & 0 \\
0 & 0 & a \\
3-6 \tanh ^{2}(\bar{x}) & -\gamma & -\mu
\end{array}\right] .
$$

We obtain the related eigenvalues $\lambda_{j}(j=1,2,3)$ by searching for the zeros of the characteristic polynomial:

$$
\mathrm{P}(\lambda)=\operatorname{det}\left(M_{J}-\lambda I_{d}\right)=\lambda^{3}+\mu \lambda^{2}+a \gamma \lambda-3 a\left(1-2 \tanh ^{2}(\bar{x})\right),
$$

where $I_{d}$ refers to the $3 \times 3$ identity matrix. From the graphs in Figure 1, we notice that the equilibrium point $E_{0}$ has its $x$ coordinate with magnitude smaller than unity. Also, the related characteristic polynomial possesses coefficients with different signs, and thus, $E_{0}$ is always unstable according to the Routh stability theorem. The stability of the pair of fixed points $\left(E_{1}\right.$ and $\left.E_{2}\right)$ changes with the values of parameters $\alpha$ and $\gamma$. From both the Routh-Hurwitz stability criterion $[1,2]$ and the Hopf bifurcation theorem, we derived the following results about the stability of the above pair of equilibrium points. Each of the fixed points $E_{n}(n=1,2)$ remains stable only for values of $\gamma>\gamma_{c}\left(x_{n}\right)=\gamma_{c n}=3\left(2 \tanh ^{2}\left(x_{n}\right)-1\right) / \mu$. If the parameter $\gamma$ is brought beyond the critical value $\gamma_{c n}$, the fixed point $E_{j}$ becomes unstable. We now investigate the Hopf bifurcation related to the fixed point $E_{n}(n=1,2)$ when $\gamma$ is considered as the bifurcation control parameter. The following analytical conditions have been derived:

$$
\begin{aligned}
\gamma_{H}\left(x_{n}\right) & =\gamma_{c n}=\frac{3\left(2 \tanh ^{2}\left(x_{n}\right)-1\right)}{\mu}, \\
\omega_{H}\left(x_{n}\right) & =\sqrt{a \gamma_{c n}}, \\
\operatorname{Re}\left(\frac{d \lambda}{\left.d \gamma\right|_{\gamma=\gamma_{c}}}\right) & =\frac{-a}{2+2 a \gamma_{c n}} \neq 0 .
\end{aligned}
$$

As a result, systems (1a) and (1b) exhibit a Hopf bifurcation from $E_{n}(n=1,2)$ when $\gamma=\gamma_{H}\left(x_{n}\right)$, and a limit cycle will develop around the point $E_{n}(n=1,2)$. Equation (7b) defines the frequency of oscillations $\left(\omega_{H}\right)$, while the transversality condition is expressed by equation (7c). As sample numerical results, both equilibrium points $E_{1}$ and $E_{2}$ undergo a Hopf bifurcation at $\gamma=\gamma_{c 1}=\gamma_{c 2} \approx 2.496$ for $k=$ 0.0 and $\mu=1.0$. In contrast, for $k=0.25$ and $\mu=1.0$, the Hopf bifurcation values are $\gamma_{c 1} \approx 2.40$ and $\gamma_{c 2} \approx 2.58$, respectively, for $E_{1}$ and $E_{2}$. From the study presented above, we conclude that both three equilibriums are unstable in any regime (periodic or chaotic oscillations) of operation, and systems (1a) and (1b) exhibit self-excited attractors accordingly $[36,37]$.

\section{Numerical Study}

3.1. Scenario to Chaos. To highlight the influence of system parameters on the dynamics of the system, we keep $a=10.0$ and use $\gamma$ and $k$ as control parameters. Figure 2 provides the bifurcation diagrams of the coordinate $x_{1}$ against $\gamma$ and related plots of largest Lyapunov exponent [38] for two different values of $k$ (i.e., $k=0.00$ and $k=0.25$ ). These diagrams are obtained by scanning the parameter downward without resetting the values of initial conditions, starting the system from the initial state $( \pm 0.5,0,0)$, respectively. We know that, for $k=0.0$ (see Figure 2), the system is symmetric as well as related dynamical structures (i.e., equilibrium points, attractors, and basins of attractions). For this particular case, it can be seen from the diagram of Figure 2 (left panel) that there exist two symmetric bifurcation branches (blue and magenta), exhibiting a period doubling sequence to chaos for decreasing $\gamma$. These branches merge at approximately $\gamma=1.0$ via the well-known symmetry recovering the crisis process. At this point, two mutually symmetric mono-scroll chaotic attractors (corresponding to the blue and magenta branches) combine to form a doublescroll strange attractor (see Figure 3). Completely different routes are found in the nonsymmetric system (i.e., $k \neq 0.0$ ). In fact, for a nonzero value of parameter $k$ (e.g., $k=0.25$ ), it can be captured from Figure 2 (right panel) that a pair of asymmetric limit cycles with different periodicity experiences each its own route of period doubling cascade to chaos when the control parameter $\gamma$ is decreased in small steps. Accordingly, blue and magenta bifurcation branches display a horizontal shift which increases with parameter $k$. For example, in the bifurcation plot of Figure 2 (right panel), the first period doubling takes place at $\gamma=0.15$ for the blue branch and $\gamma=0.25$ for the red one. Here, the merging process never occurs. Instead, the series of period doublings of coexisting asymmetric cycles yields an asymmetric double-scroll strange attractor (see Figure 4). Moreover, most fascinating properties of the asymmetric system is the sudden disappearance (via a critical transition) of one of the bifurcation branches (i.e., the magenta branch; see Figure 2) when decreasing the control parameter $\gamma$ for any nonzero value of $k$. Past this crisis event, the system experiences a single attractor that metamorphoses to an asymmetric double-scroll chaotic attractor as $\gamma$ is further decreased.

3.2. Coexistence of Multiple Attractors. The coexistence of multiple stable states [10] is one of the most attractive properties the jerk system considered in this work. This intriguing feature has been deeply investigated for the symmetric system (i.e., $k=0.00$ ) in the reference work [18]. In this section, we investigate the impact of the excitation term $(k)$ on the mechanism governing the generation of multiple solutions. In this regard, several bifurcation plots are produced following appropriate numerical techniques [18] in order to track parameter domains corresponding to the presence of multiple coexisting stable states. As sample results, Figure 5 shows the bifurcation diagrams of local maxima of $x_{1}$ variable, obtained when varying parameter $a$ 


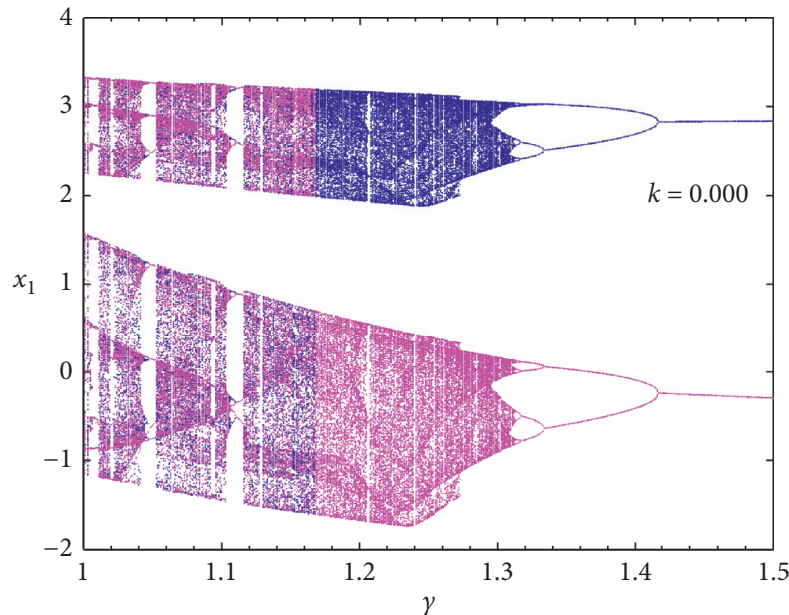

(a)

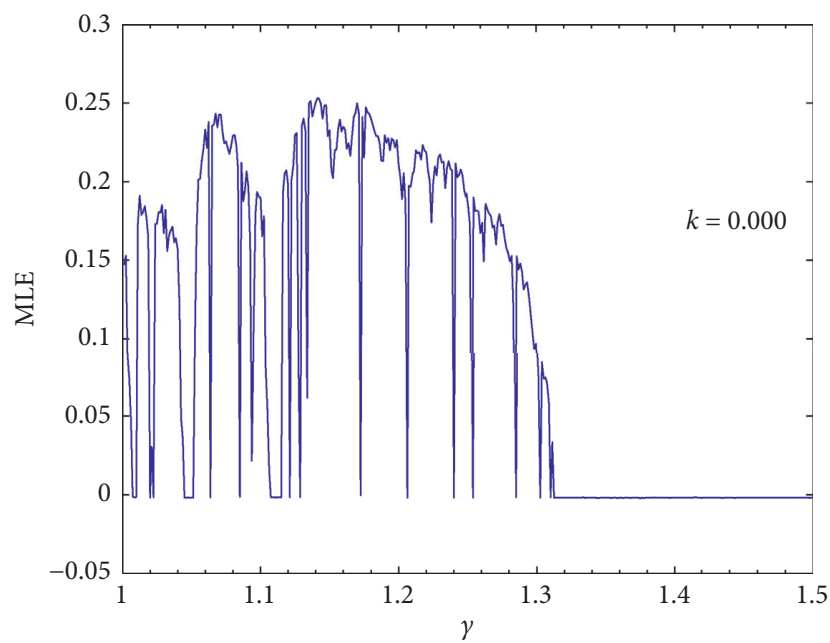

(c)

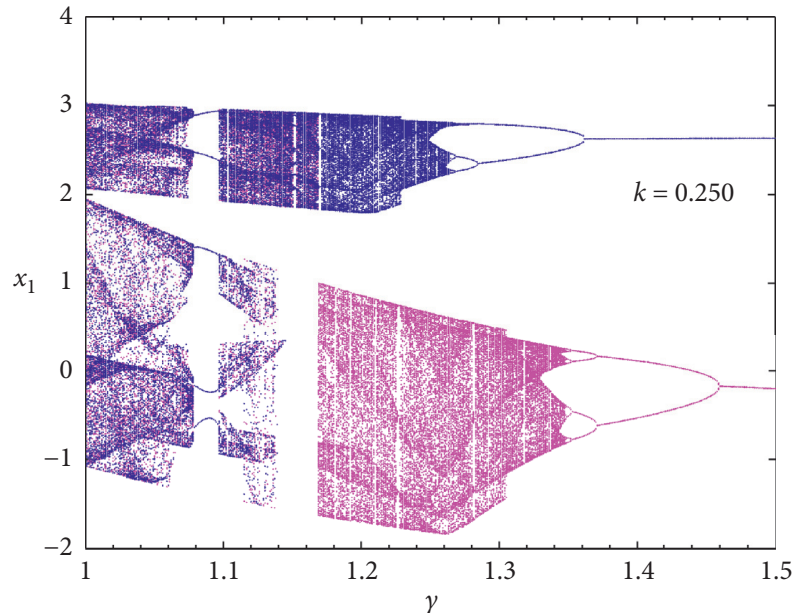

(b)

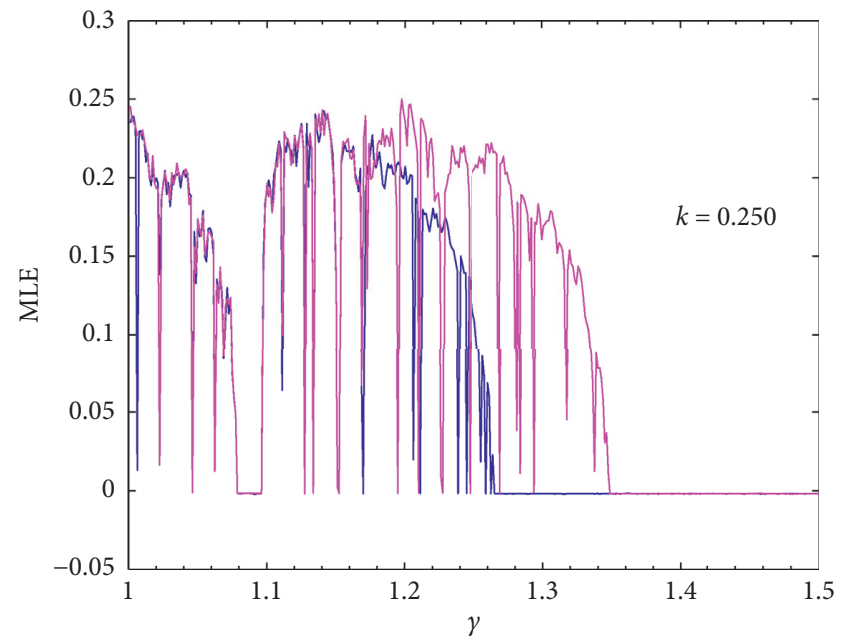

(d)

Figure 2: Bifurcation diagrams (a,b) of the system showing local maxima of the coordinate $x_{1}$ versus parameter $\gamma$ computed for $a=5$ both for the symmetric $(k=0.000)$ and the asymmetric $(k=0.250)$ modes of oscillations. The corresponding graph of maximal Lyapunov exponent is shown in the lower panel $(c, d)$.

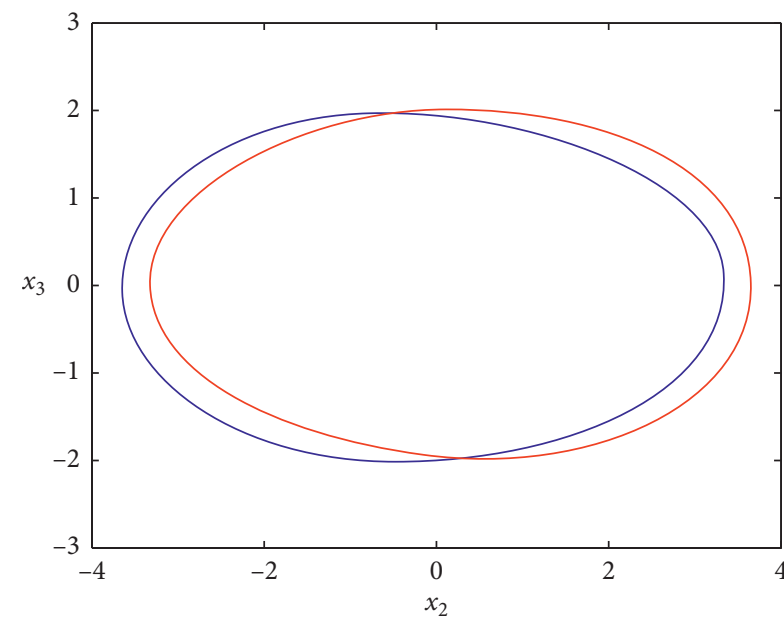

(a)

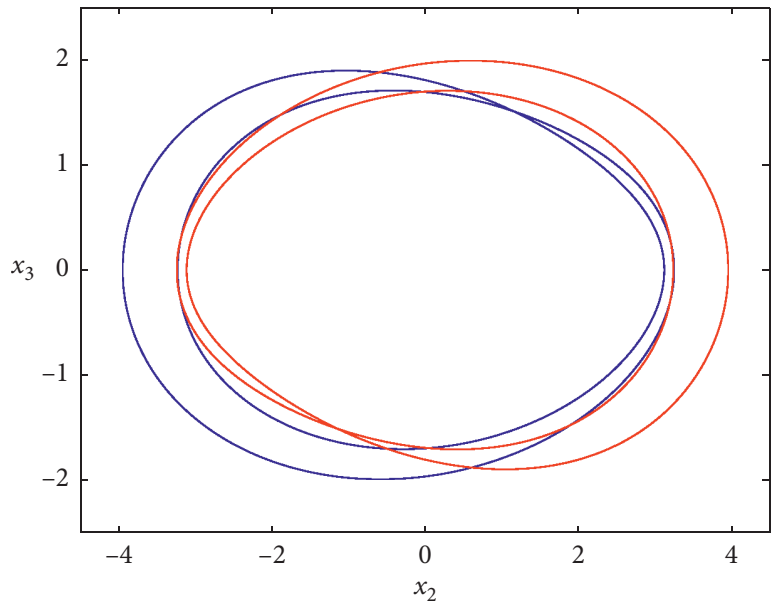

(b)

FIgURE 3: Continued. 


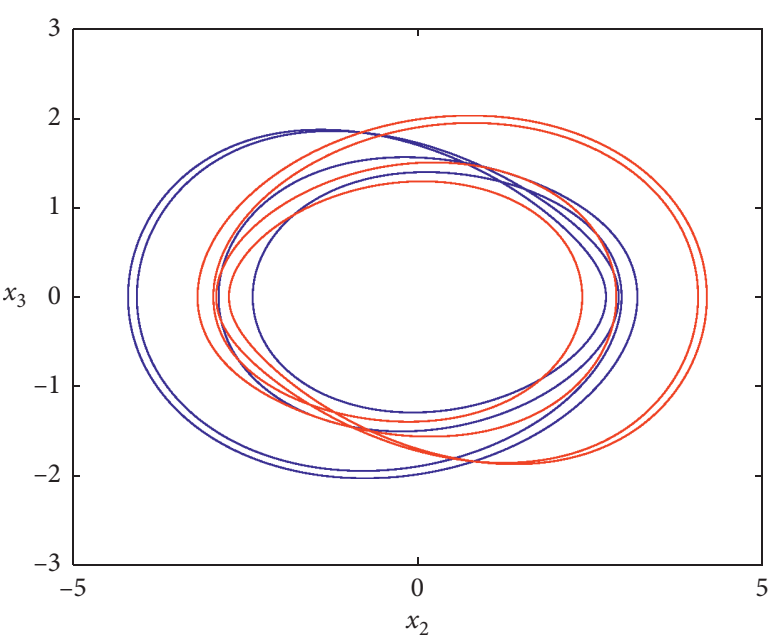

(c)

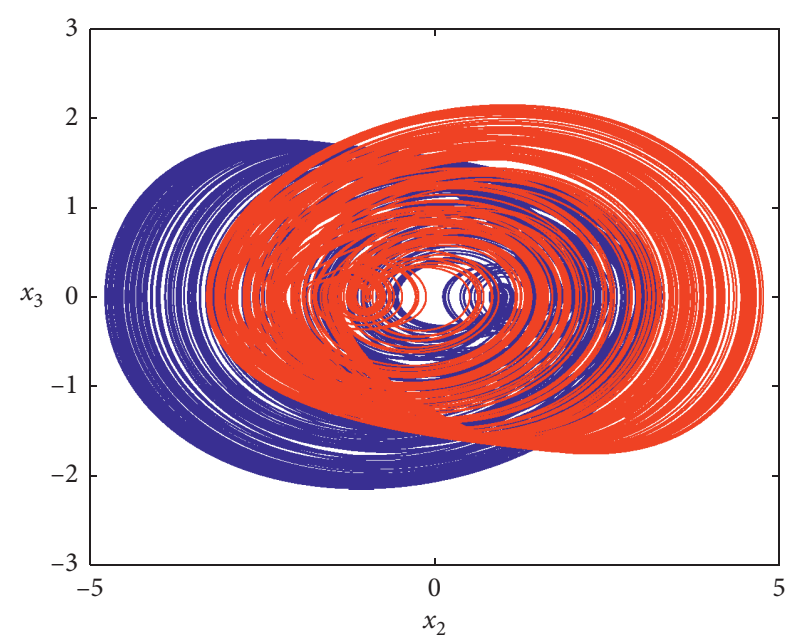

(d)

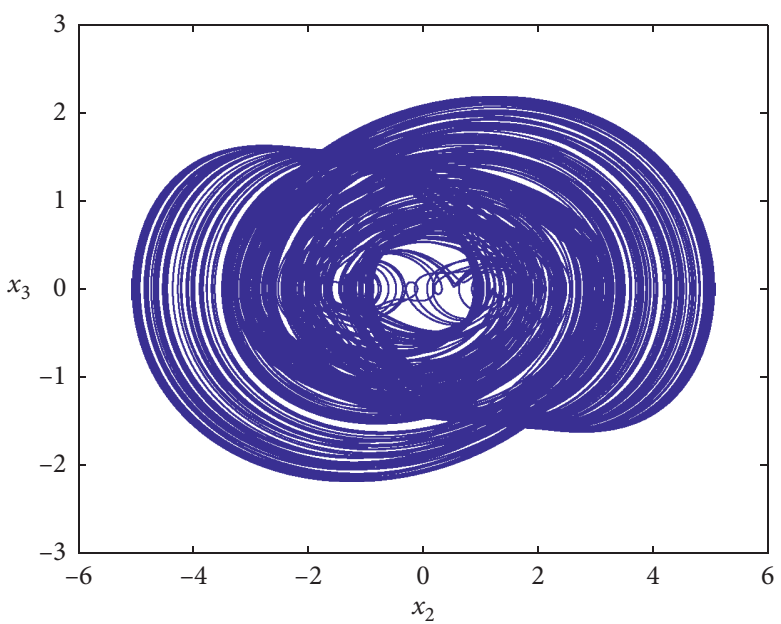

(e)

FIGURE 3: Sample chaotic phase portraits of the symmetric system: (a) a pair of period-1 cycles for $\gamma=1.6$; (b) a pair of period-2 cycles for $\gamma=1.4$; (c) a pair of period-4 cycles for $\gamma=1.323$; (d) two mutually symmetric spiraling strange attractors for $\gamma=1.18$; (e) a double-scroll strange attractor for $\gamma=1.078$. Initial conditions are not critical but fixed as $(x(0), y(0), z(0))=(1,0,0)$. The rest of parameters are $k=0.0$, $a=5.0$, and $\mu=1.0$.

in the range $5 \leq a \leq 30$ for four discrete values of $k$. Details of numerical procedures employed to produce these plots are described in Table 1. These diagrams display the intricate phenomenon of parallel branches and hysteresis which justify the occurrence of multiple attractors for several parameter sets. More importantly, notice that the merging process does occur for the symmetric system (i.e., $k=0.00$ ). In contrast, one of the branches undergoes a critical transition and collapses for nonzero values of parameter $k$ when the control parameter $\gamma$ is slowly decreased. The numerical techniques used to obtain those diagrams are provided in Table 1. A close examination of Figure 5 reveals that various combinations of coexisting attractors can be obtained when suitably selecting the system parameters. For example, Figure 6 presents the coexistence of two different chaotic attractors $(\mathrm{a}, \mathrm{b})$ computed for $a=15.0$ using two different values of initial conditions. The corresponding cross section of the basins of attraction is provided in (c) using the same colors as the relevant attractors. In this figure, the red zone indicates unbounded dynamics. In the same line, Figure 7 depicts three different asymmetric coexisting attractors and corresponding cross sections of the basin of attraction. More interestingly, by choosing appropriately the values of system parameters and initial conditions, four asymmetric coexisting attractors can be found as exemplified in Figure 8. A more intriguing situation is depicted in Figure 9 where up to five different asymmetric attractors coexist. The corresponding bifurcation like sequence of local maxima of the coordinate $x_{1}$ versus initial condition $x_{1}(0)$ is provided in the graph of Figure 9 (f). The cases reported above relate to the asymmetric mode of operation, and a different configuration of coexisting attractors occurs in the symmetric regime where coexisting attractors now appear only in mutually symmetric pairs (see Figure 10). 


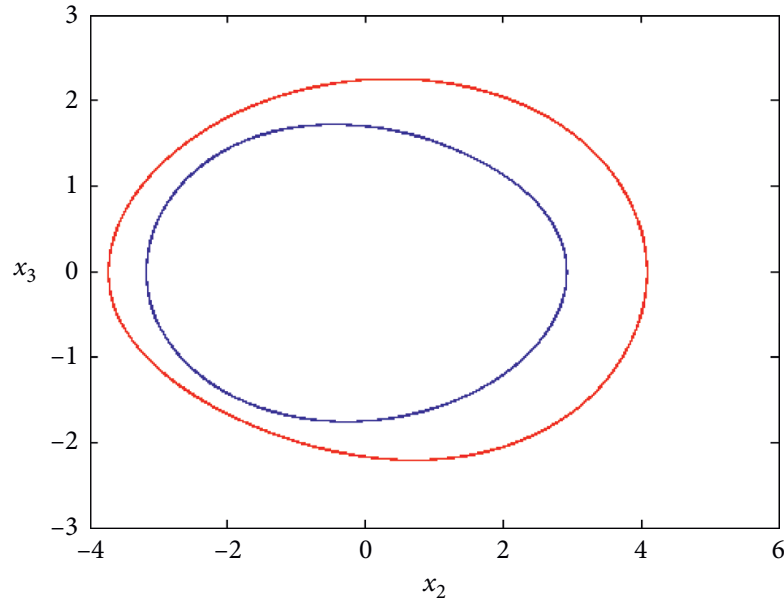

(a)

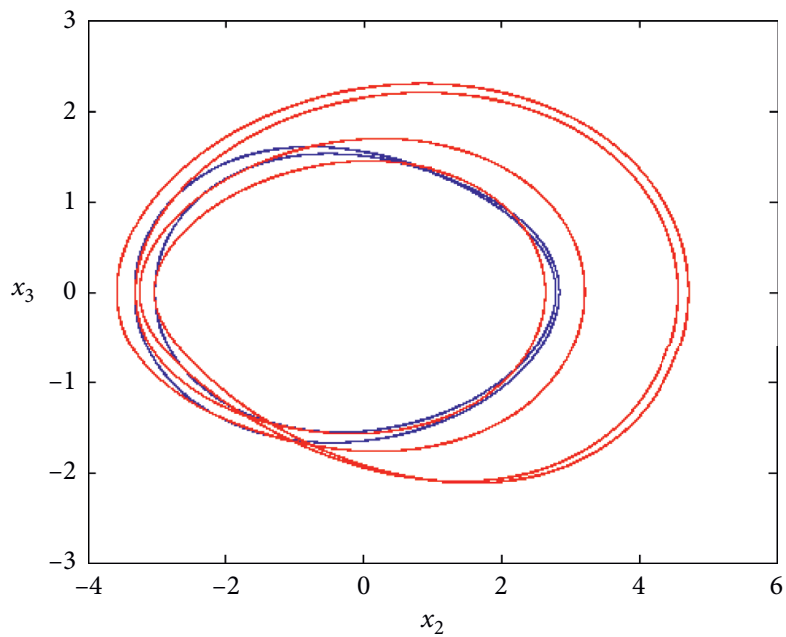

(c)

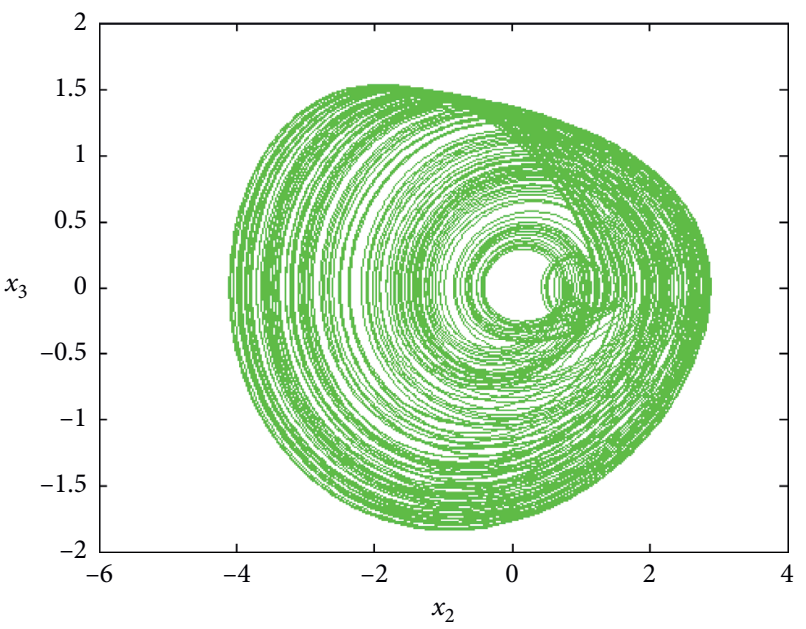

(e)

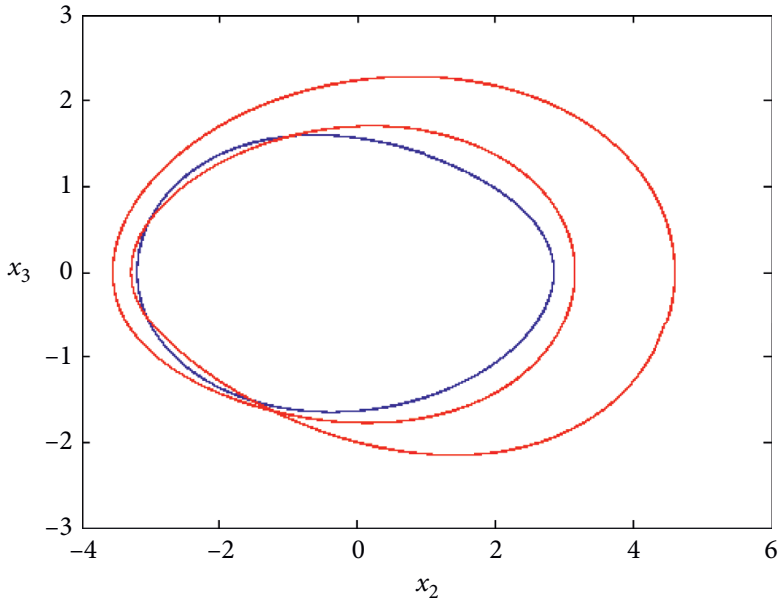

(b)

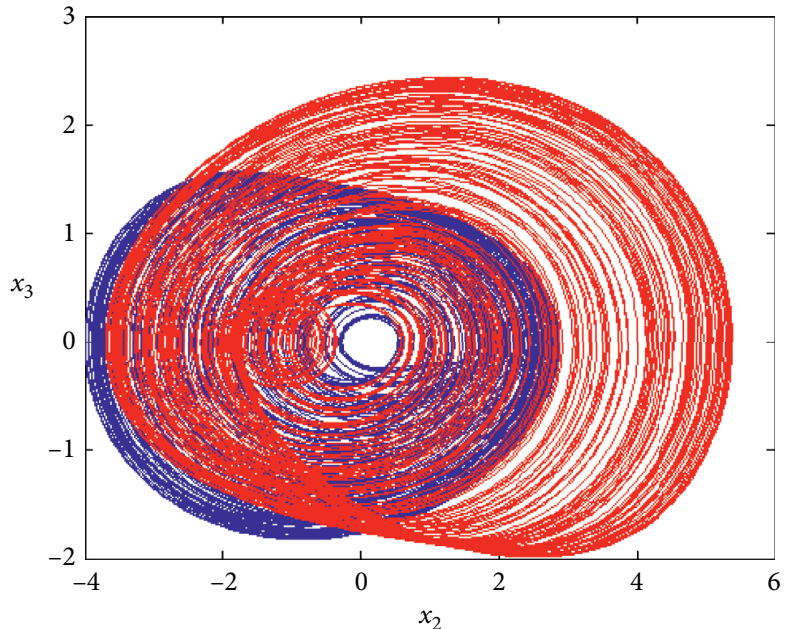

(d)

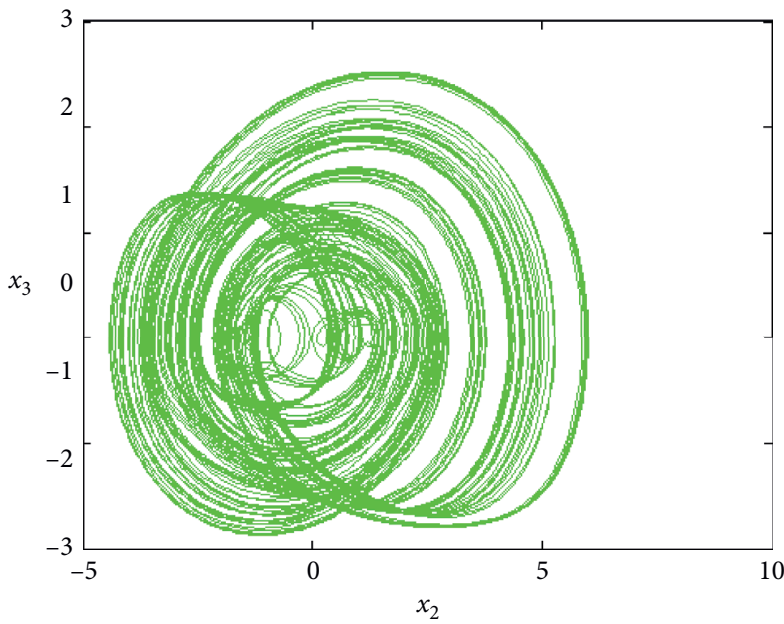

(f)

Figure 4: Computer-generated phase space trajectories of the system projected onto the $y-z$ planes obtained for some discrete values of parameter $\gamma$ : (a) coexistence of two period-1 limit cycles for $\gamma=1.6$; (b) coexistence of period-1 and period-2 cycles for $\gamma=1.4$; (c) coexistence of period-4 and period-2 cycles for $\gamma=1.359$; (d) coexistence of two nonsymmetric strange attractors for $\gamma=1.2$; (e) a nonsymmetric strange attractor for $\gamma=1.16$; (f) an asymmetric double-scroll strange attractor for $\gamma=1.0$. The computations are done with $k=0.25, \mu=1.0$, and $a=5.0$. Initial conditions are $(x(0), y(0), z(0))=( \pm 1,0,0)$. 


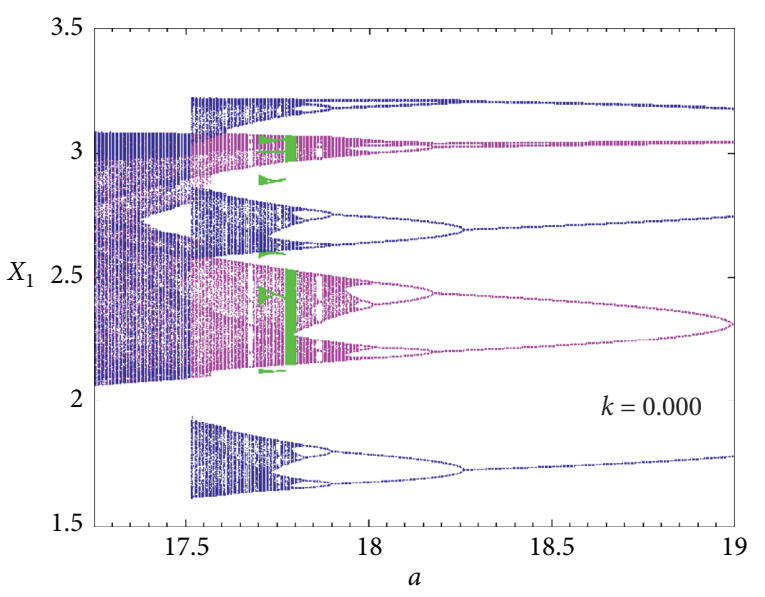

(a)

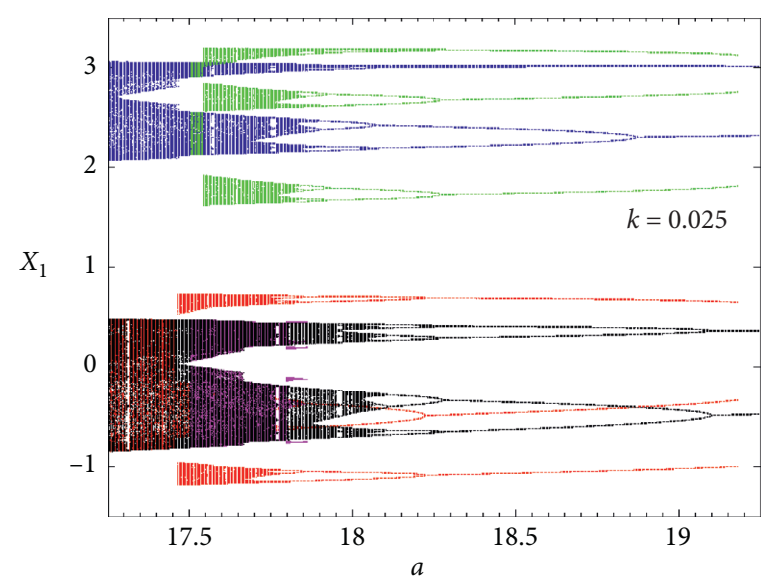

(b)

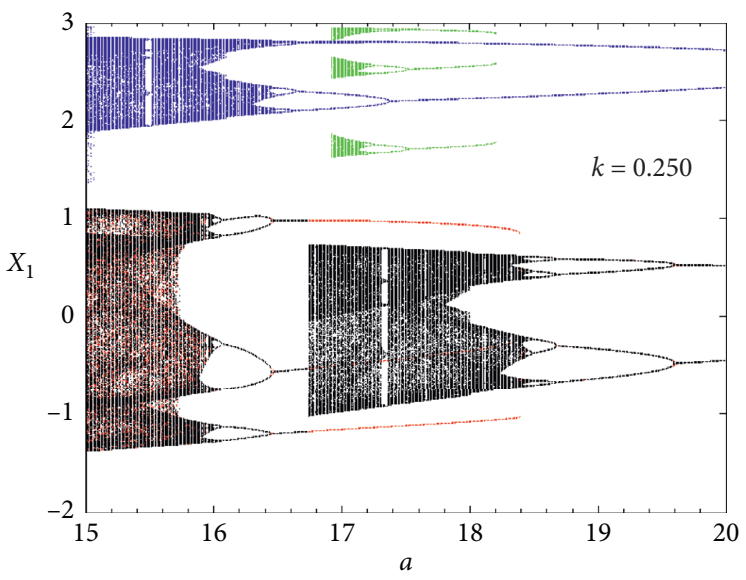

(c)

FIGURE 5: Enlargements of the bifurcation diagrams of the system showing various coexisting bifurcation branches and hysteresis, computed for three different values of parameterk, namely, $k=0.000, k=0.025$, and $k=0.250$. Detail numerical procedures used to obtain those diagrams are provided in Table 1. The rest of parameters are same as in Figure 5.

TABLE 1: Detailed numerical procedures used to obtain the bifurcation diagrams of Figure 5.

\begin{tabular}{|c|c|c|c|c|}
\hline Graph identification & Color data & Parameter range & Sweeping direction & $\begin{array}{c}\text { Initial condition } \\
\left(x_{1}(0), x_{2}(0), x_{3}(0)\right)\end{array}$ \\
\hline \multirow{3}{*}{$k=0.000$} & Magenta & $17.25 \leq a \leq 19$ & Upward & $(0.1,0,0)$ \\
\hline & Green & $17.70 \leq a \leq 19$ & Upward & $(0.2,0,0)$ \\
\hline & Blue & $5 \leq a \leq 19$ & Downward & $(0.6,0,0)$ \\
\hline \multirow{5}{*}{$k=0.025$} & Blue & $17.25 \leq a \leq 19.25$ & Upward & $(+2,0,0)$ \\
\hline & Red & $17.5 \leq a \leq 19.18$ & Downward & $(-2,0,0)$ \\
\hline & Black & $17.25 \leq a \leq 19.25$ & Upward & $(-5,0,0)$ \\
\hline & Magenta & $17.25 \leq a \leq 17.86$ & Downward & $(-0.8,0,0)$ \\
\hline & Green & $17.5 \leq a \leq 19.18$ & Downward & $(1,0,0)$ \\
\hline \multirow{4}{*}{$k=0.250$} & Blue & $15 \leq a \leq 20$ & Downward & $(5,0,0)$ \\
\hline & Black & $15 \leq a \leq 20$ & Downward & $(-5,0,0)$ \\
\hline & Red & $15 \leq a \leq 20$ & Upward & $(-5,0,0)$ \\
\hline & Green & $16.92 \leq a \leq 18.20$ & Upward & $(3,0,0)$ \\
\hline
\end{tabular}

From the above investigations, we notice that the occurrence of multiple attractors is possible both in the unforced (i.e., $k=0.0)$ and the forced $(k \neq 0.0)$ regimes. The latter situation is being much more challenging for analysis provided that the occurrence of multiple solutions cannot be explained based on symmetry arguments. At this point, we would like to stress that the occurrence of multiple attractors has reported numerous other problems from diverse fields of science and technology. This feature can be advantageously exploited in engineering applications such as image 


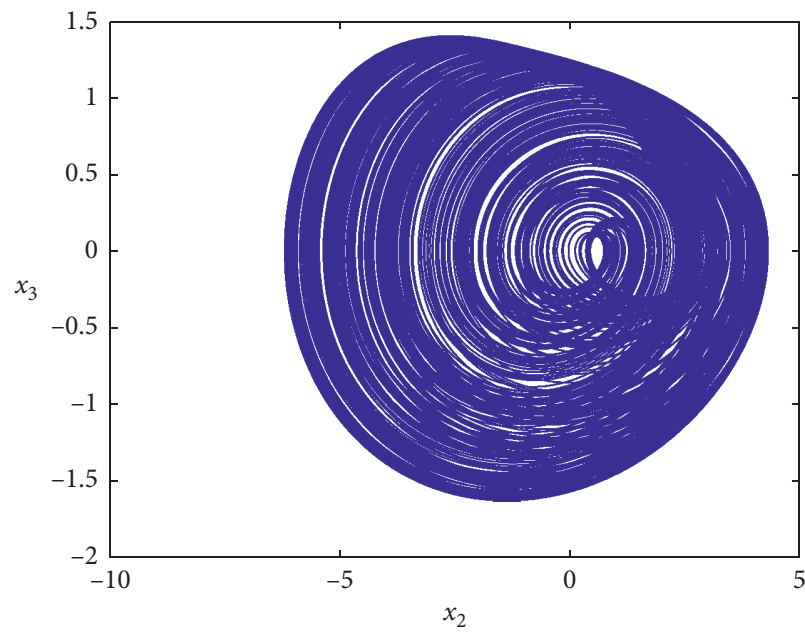

(a)

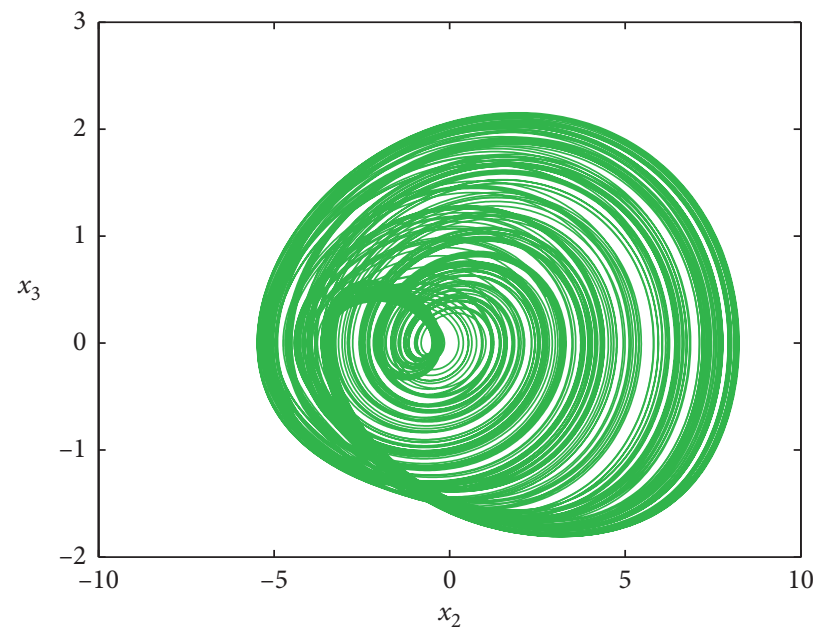

(b)

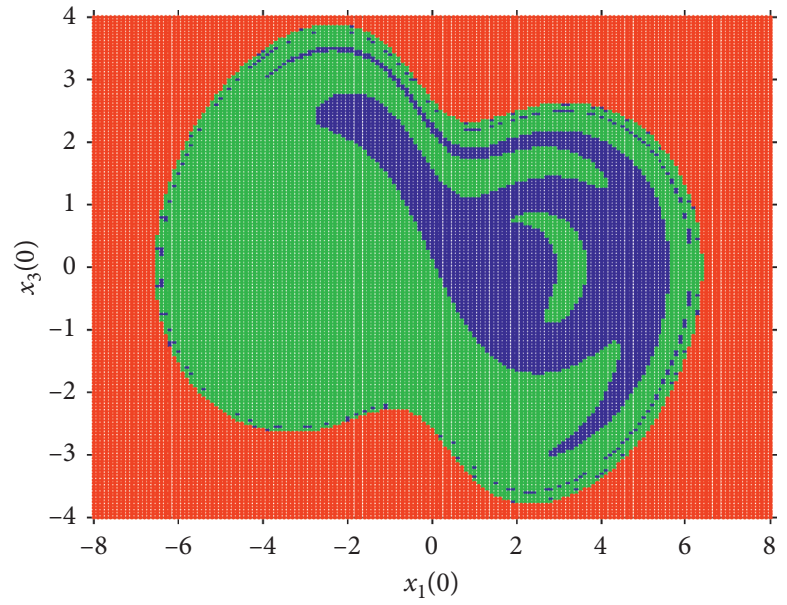

(c)

Figure 6: Coexistence of two different attractors for $a=12.0, \gamma=1.0, \mu=0.95$, and $k=0.25$. Initial conditions $\left(x_{1}(0), x_{2}(0), x_{3}(0)\right)$ are $(0.4,0,0)$ and $(-0.4,0,0)$ for $(a)$ and (b), respectively. The corresponding cross section of the basins of attraction is provided in (c) using the same colors as the relevant attractors. The red zone denotes unbounded dynamics.

encryption and random signal generation as well. However, in cases where only a single stable attractor is desired, some control strategies may be developed. Detailed analysis concerning this point is out of the scope of this paper. Accordingly, interested readers may obtain precious information from the review work of [10].

3.3. Coexisting Bubbles of Bifurcation. Another interesting and striking event revealed monitoring the parameters of system (1) is the phenomenon of antimonotonicity [39-44]. In fact, the period doubling transition to chaos followed by the reverse bifurcation scenario is found when varying the control parameter $a$ for several values of $\gamma$ in case of a zero forcing term (i.e., $k=0.0$ ). Sample results are depicted in Figure 11 which presented five bifurcation plots of the coordinate $x$ versus $a$ for five discrete values of $\gamma$. In each case of the diagrams in Figure 11, there are two symmetric bifurcation diagrams due to the symmetry of the model. From Figure 11, we note that a period-1 bubble is obtained for $\mu=1.0$. As $\gamma$ decreases the sequence $P_{1}$ bubble $\longrightarrow P_{2}$ bubbles $\longrightarrow P_{4}$ bubble $\longrightarrow P_{8}$ bubbles $\longrightarrow$ full, Feigenbaum tree takes place. This behavior corresponds to the symmetric system $(k=0.0)$ and much more complex nonlinear dynamics arise in the presence of a nonzero excitation force (i.e., $k \neq 0.0$ ), as exemplified in Figure 12. This latter figure depicts the bifurcation plots of the coordinate $x$ against parameter $a$ obtained for several discrete values of $k$ while maintaining $\gamma=1.475$ and $\mu=0.875$. In contrast to the situation presented in Figure 11, lower and upper bifurcation branches are nonsymmetric, depicting a horizontal shift and exhibiting different periodicities. This striking behavior (engendered by symmetry break) is rarely reported and thus represents an enriching contribution to the behavior of these types of systems.

\section{Control of Multistability}

Recently, a control method referred to as linear augmentation is described which is suitable to control the dynamics 


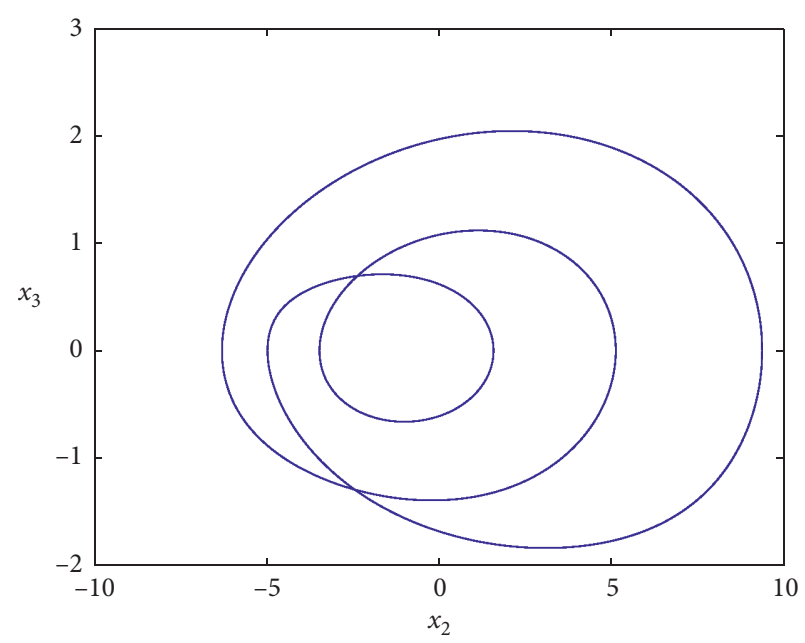

(a)

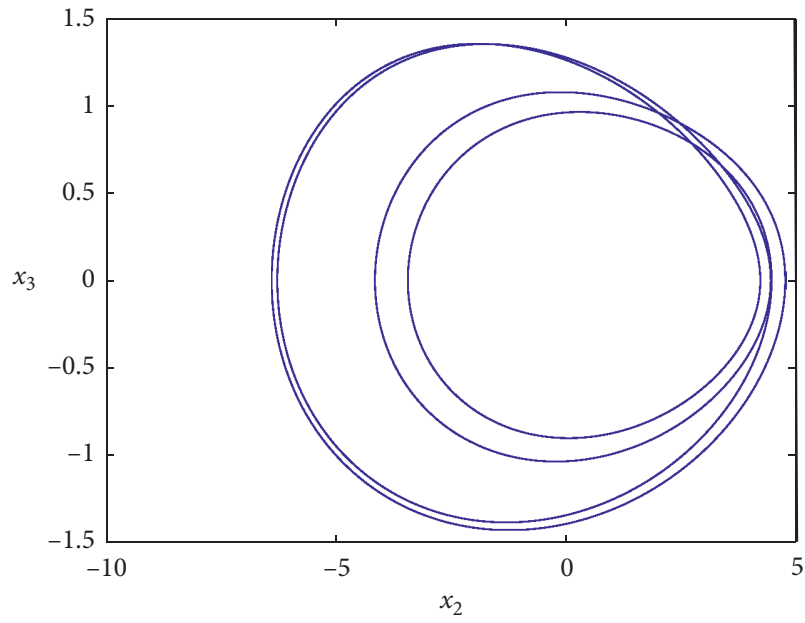

(c)

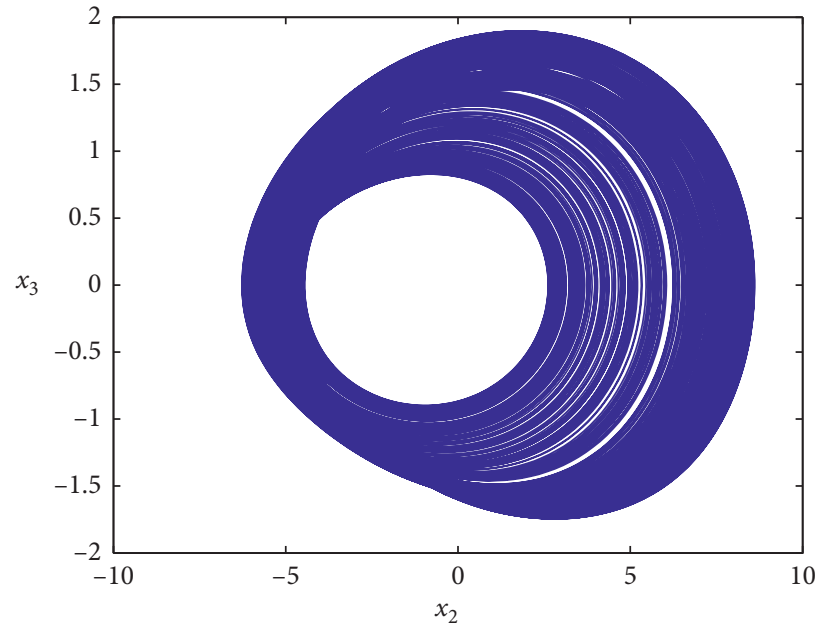

(b)

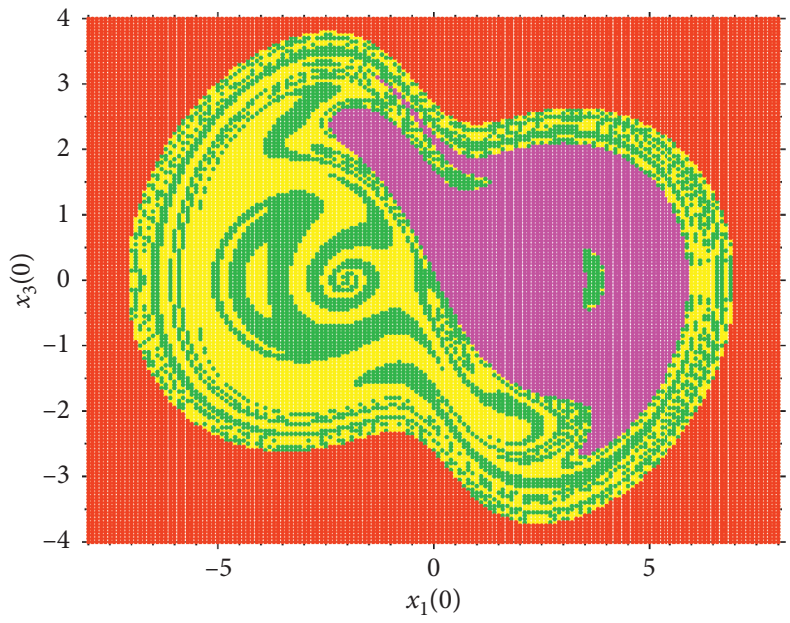

(d)

Figure 7: Coexistence of three different attractors for $a=16.85, \gamma=1.0, \mu=0.95$, and $k=0.25$. Initial conditions $\left(x_{1}(0), x_{2}(0), x_{3}(0)\right)$ are $(-1.8,0,0)$ for attractor in (a), $(-1.6,0,0)$ for attractor in $(b)$, and $(0.4,0,0)$ for attractor in (c). Green, magenta, and yellow colors correspond to the attractors in (a), (b), and (c), respectively. The red zone corresponds to unbounded dynamics.

of a nonlinear system without perturbing the system's parameters [45-47]. In this strategy, a nonlinear dynamic system is coupled with a linear one. The motivations of this coupling scheme are twofold: (a) to stabilize the steady state in a given nonlinear oscillator; (b) to adjust the number of coexisting attractors for a multistable system. Accordingly, the dynamics of the jerk system with hyperbolic tangent nonlinearity coupled to a linear system is described by the following fourth-order system:

$$
\left\{\begin{array}{l}
\dot{x}_{1}=x_{2}, \\
\dot{x}_{2}=a x_{3}, \\
\dot{x}_{3}=-\gamma x_{2}-\mu x_{3}-k-3 x_{1}+6 \tanh \left(x_{1}\right)+\delta u, \\
\dot{u}=-\sigma u-\delta(x-\beta) .
\end{array}\right.
$$

Here, $\sigma \geq 0$ represents the decay parameter of the linear system $u, \delta$ denotes the coupling strength, and $\beta$ is the control parameter, which serves to locate the position of the equilibrium points. For $\delta=0$, both oscillators evolve independently, and the linear system exhibits a fixed-point motion. For a nonzero coupling coefficient (i.e., $\delta \neq 0$ ), there is a mutual influence between the nonlinear oscillator and the linear system resulting in the symmetry breaking of the whole system even for $k=0.0$. The fixed points of the coupled system are yielded by the following nonlinear algebraic system:

$$
\left\{\begin{array}{l}
-k-3 x_{1}+6 \tanh \left(x_{1}\right)+\delta u=0 \\
-\sigma u-\delta(x-\beta)=0
\end{array}\right.
$$

System (9) clearly shows that the fixed points are asymmetrically located in state space, and their number strongly depends on the values of the linear system parameters as well as the coupling strength. Considering the case where the system develops six distinct periodic and chaotic attractors, we examine the range of coupling parameter corresponding to a monostable behavior of the 


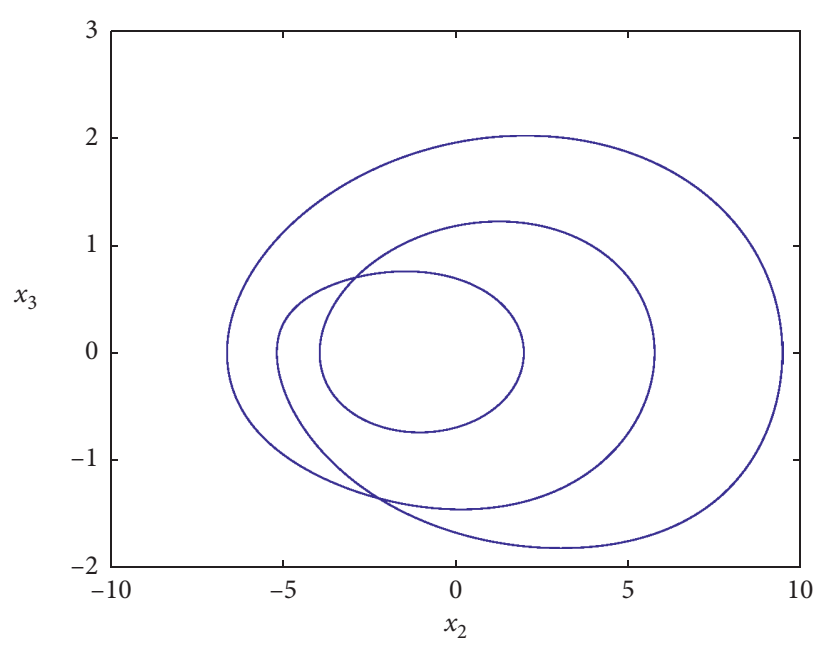

(a)

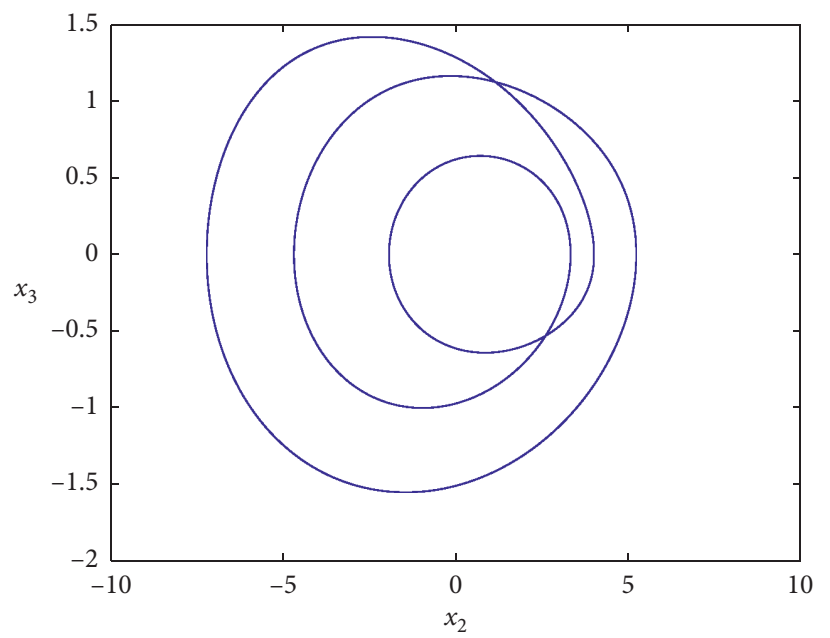

(c)

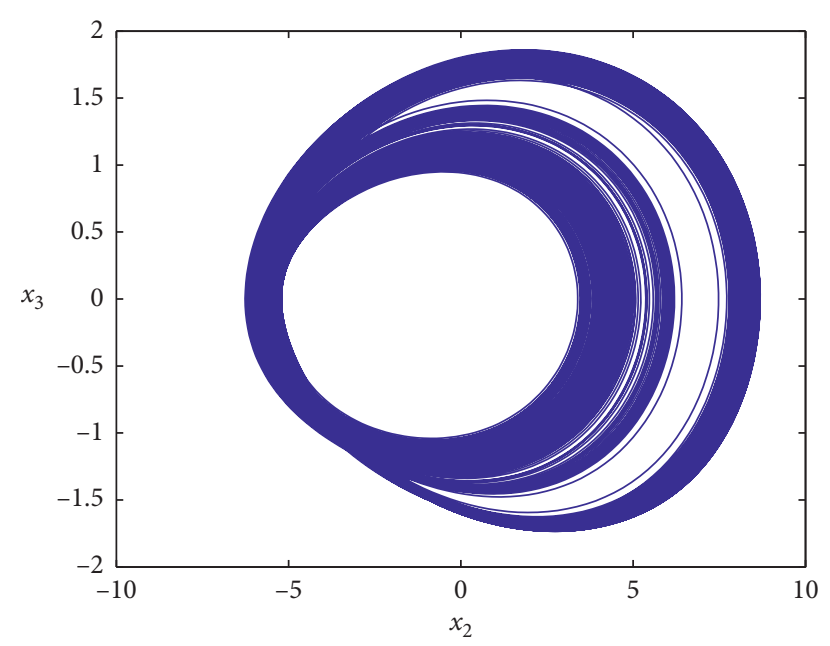

(b)

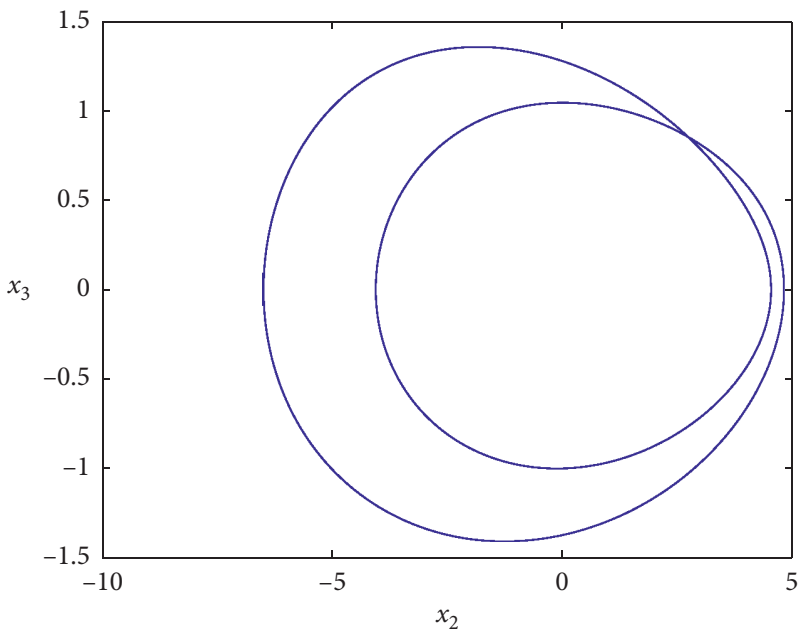

(d)

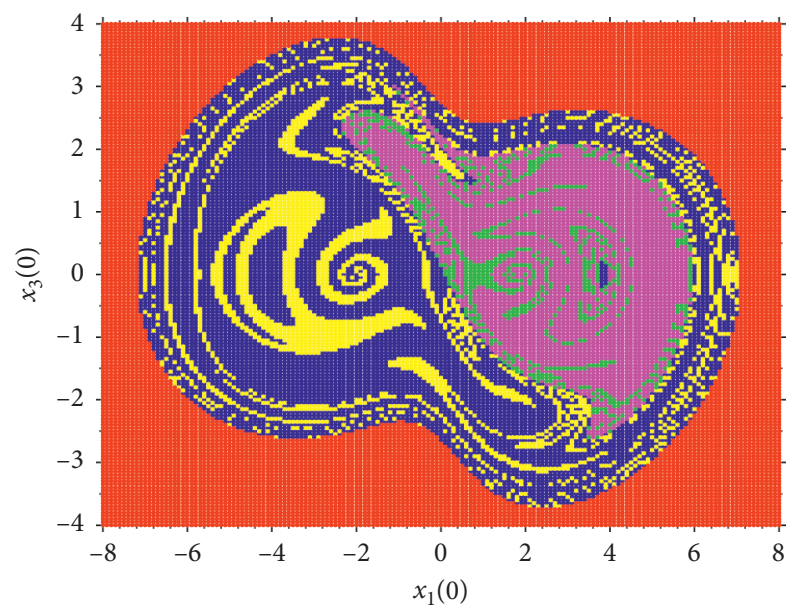

(e)

Figure 8: Coexistence of four different attractors for $a=18.0, \gamma=1.0, \mu=0.95$, and $k=0.25$. Initial conditions $\left(x_{1}(0), x_{2}(0), x_{3}(0)\right)$ are $(-1.8,0,0)$ for the period-3 cycle in (a) with blue basin, $(-1.6,0,0)$ for the chaotic attractor in (b) with green basin, $(0.2,0,0)$ for attractor in (c) with magenta basin, and $(0.4,0,0)$ for attractor in (d) with yellow basin. (e) Red zones indicate unbounded dynamics. 


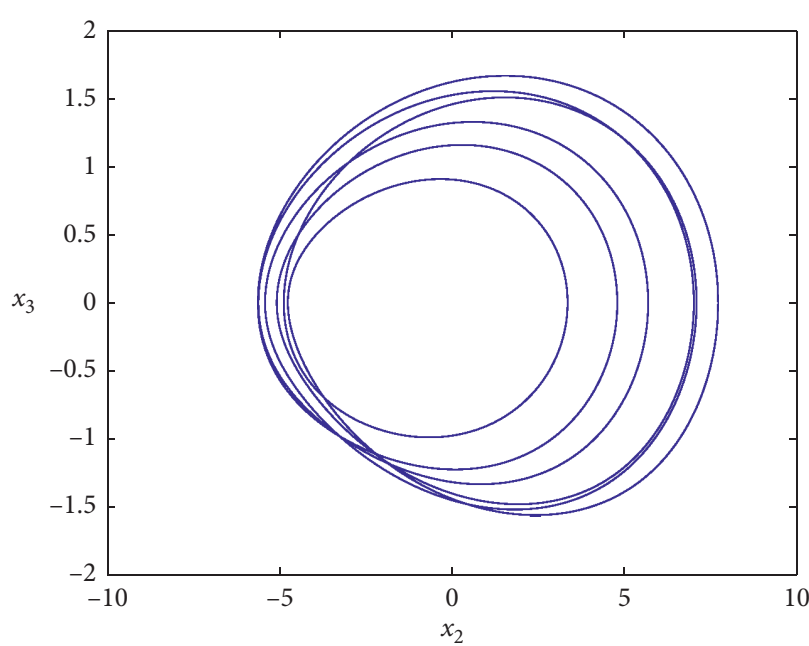

(a)

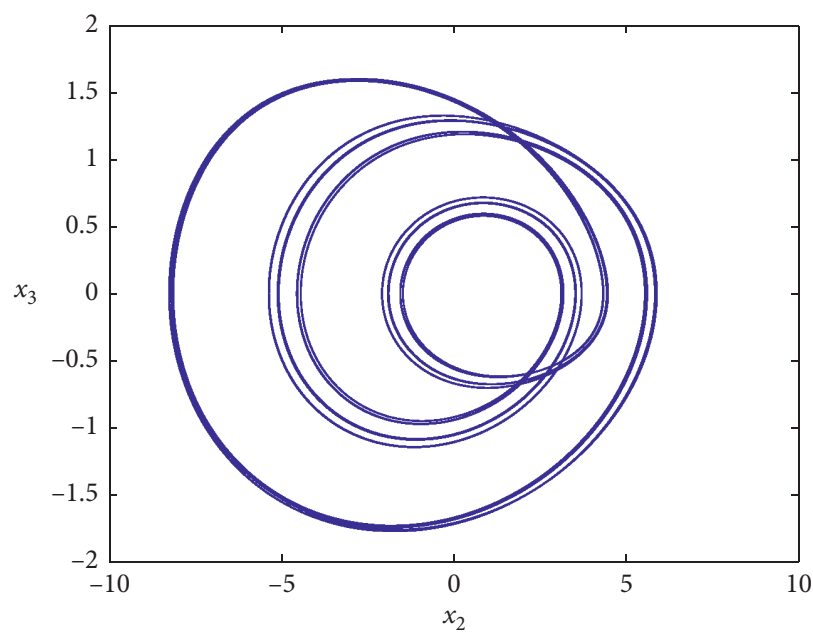

(c)

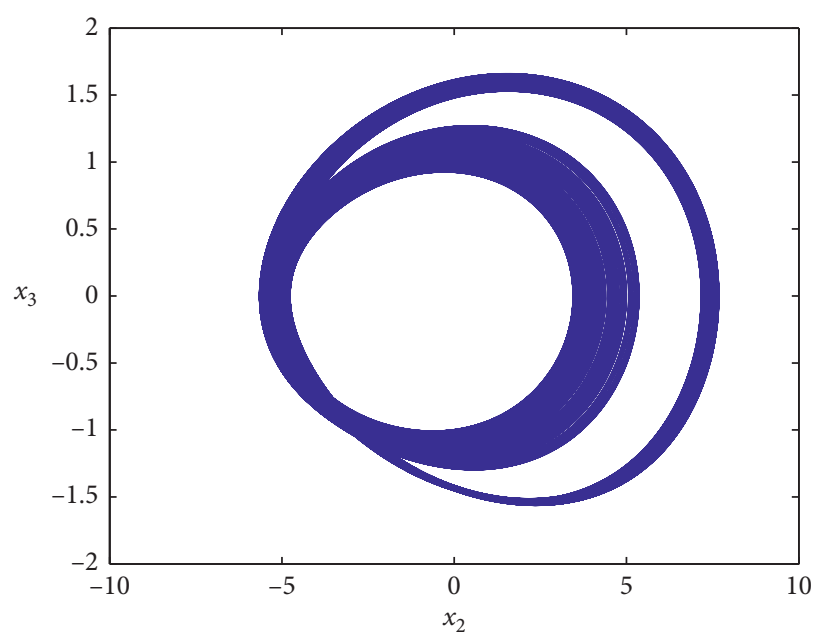

(e)

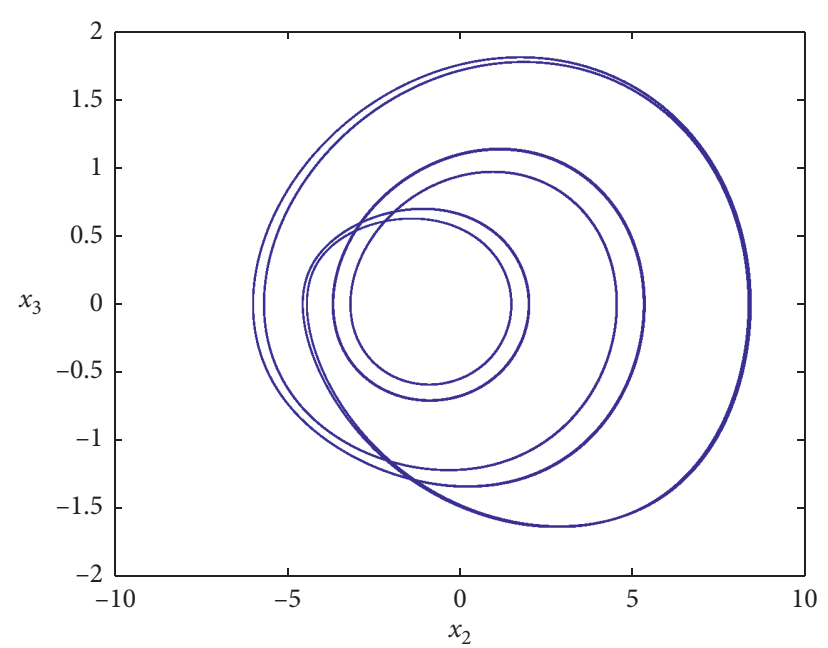

(b)

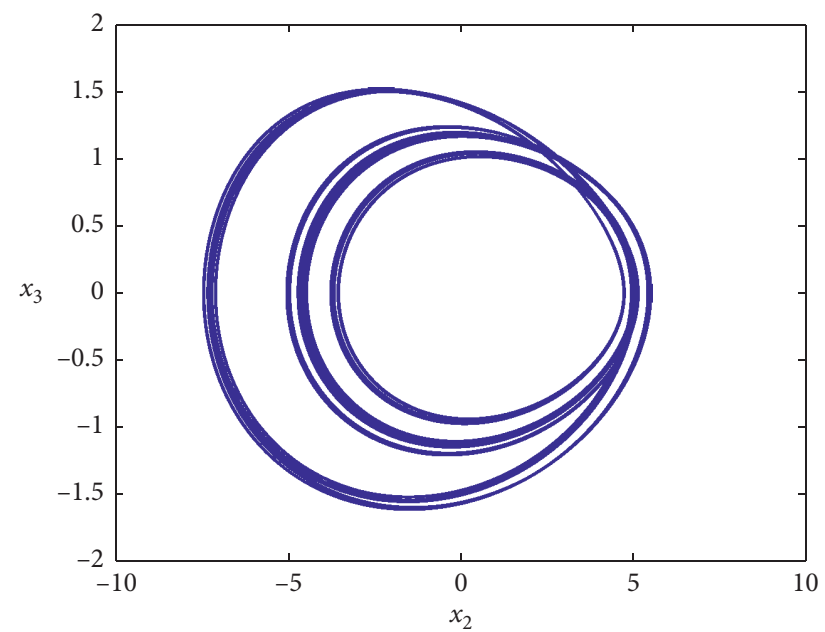

(d)

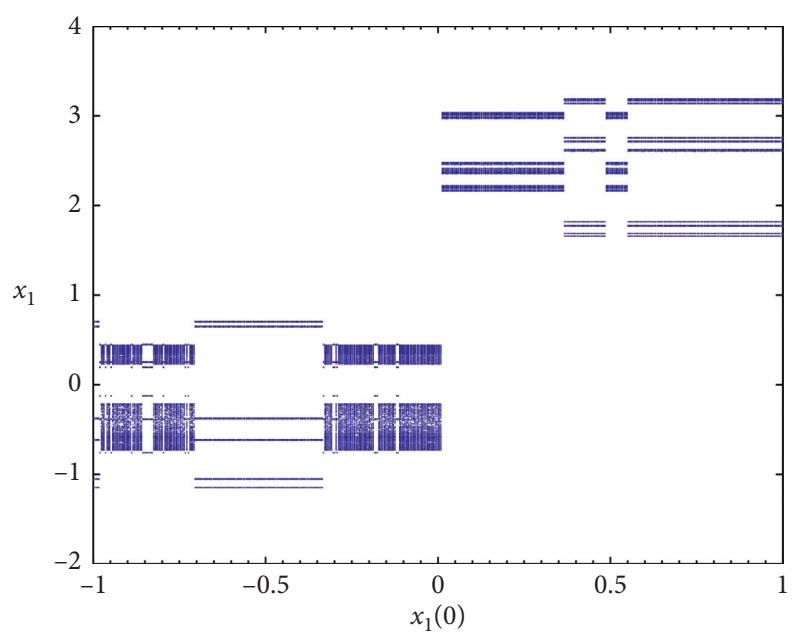

(f)

Figure 9: Coexistence of five different attractors for $a=17.85, \gamma=1.0, \mu=0.95$, and $k=0.025$. Initial conditions $\left(x_{1}(0), x_{2}(0), x_{3}(0)\right)$ are $(-0.12,0,0)$ for $(a),(-1.12,0,0)$ for $(b),(0.648,0,0)$ for $(c),(0.128,0,0)$ for $(d)$, and $(-0.048,0,0)$ for $(e)$. The bifurcation-like sequence of local maxima of the coordinate $x_{1}$ versus initial condition $x_{1}(0)$ obtained with $\left(x_{1}(0), x_{2}(0)\right)=(0,0)$ is provided in graph in $(\mathrm{f})$. 


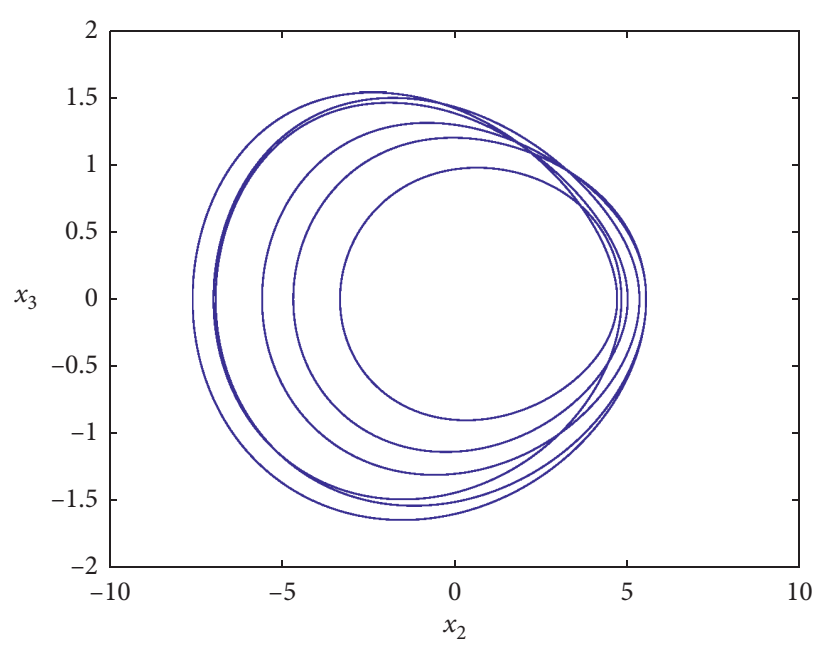

(a)

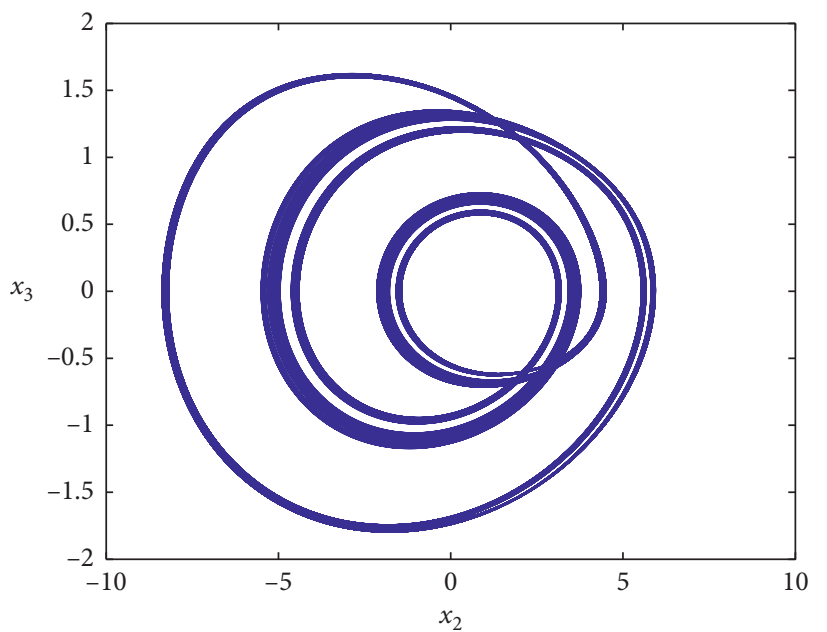

(c)

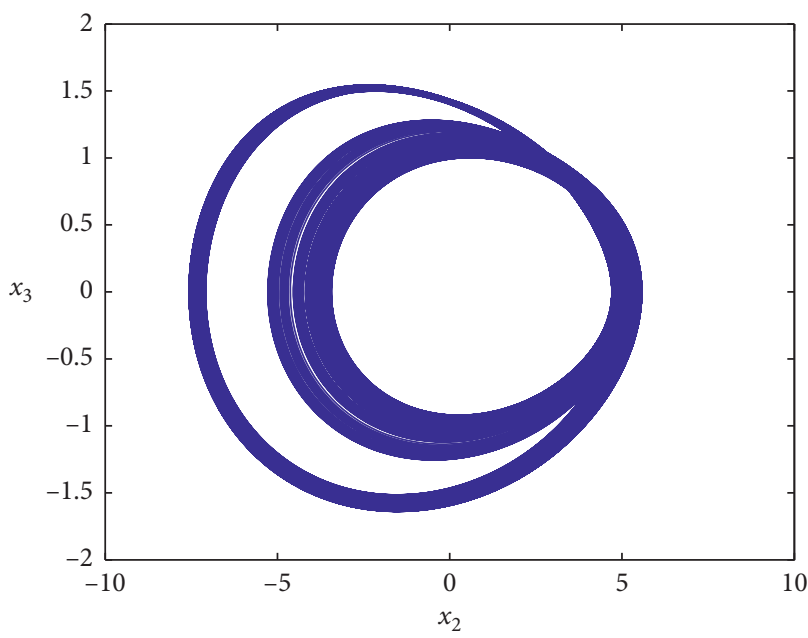

(e)

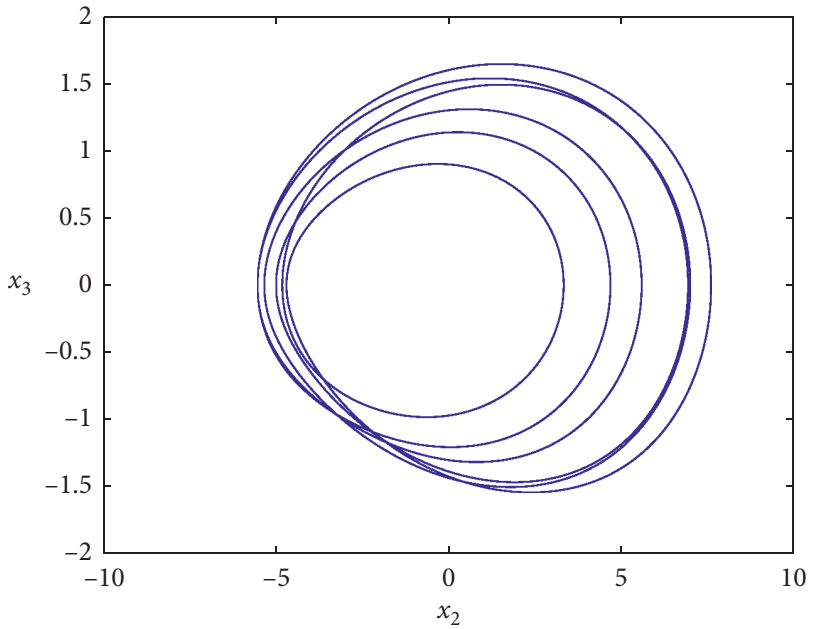

(b)

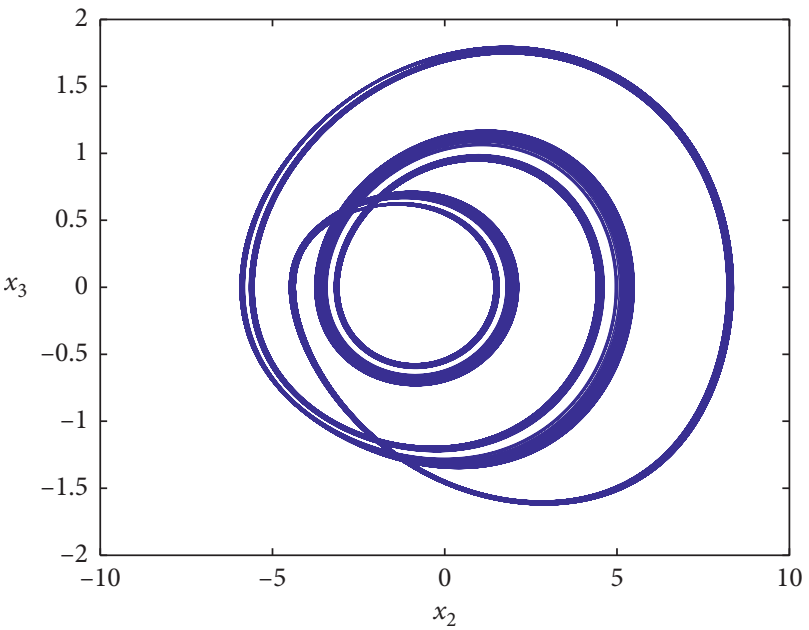

(d)

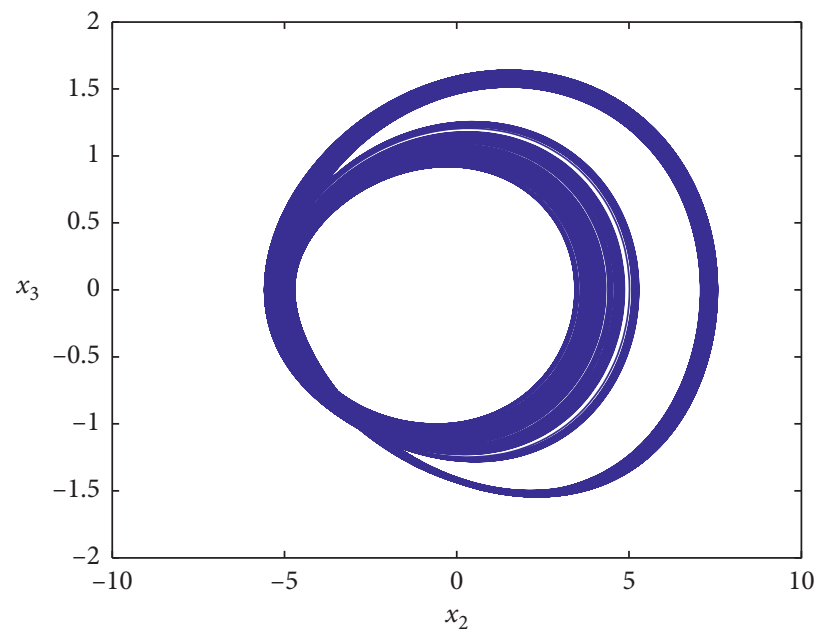

(f)

Figure 10: Coexistence of six different attractors for $a=17.77, \gamma=1.0, \mu=0.95$, and $k=0.0$. Initial conditions $\left(x_{1}(0), x_{2}(0), x_{3}(0)\right)$ are $( \pm 0.104,0,0)$ for the pair of period- 6 cycles $(a, b),( \pm 0.4,0,0)$ for the pair of three-band chaotic attractors $(c, d)$, and $( \pm 0.2,0,0)$ for twoband chaotic attractors $(e, f)$. 


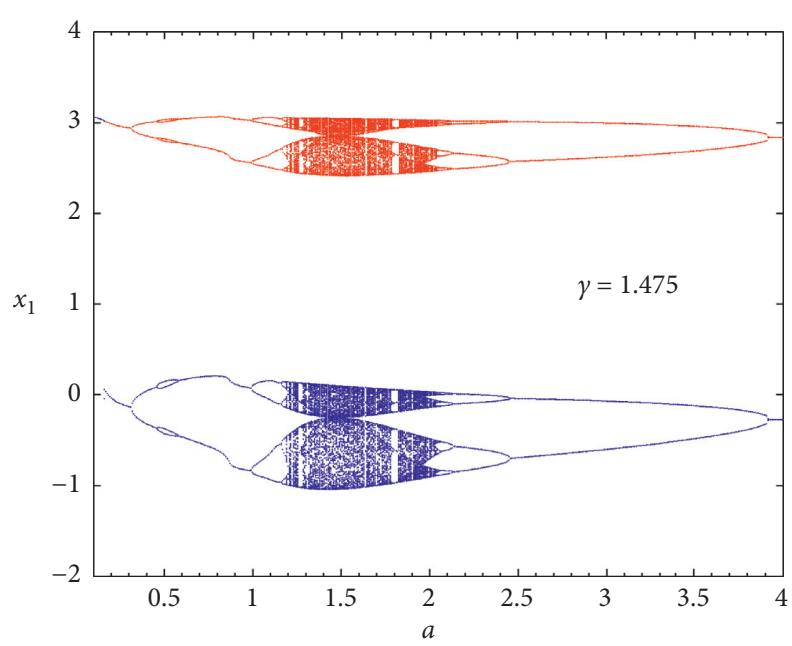

(a)

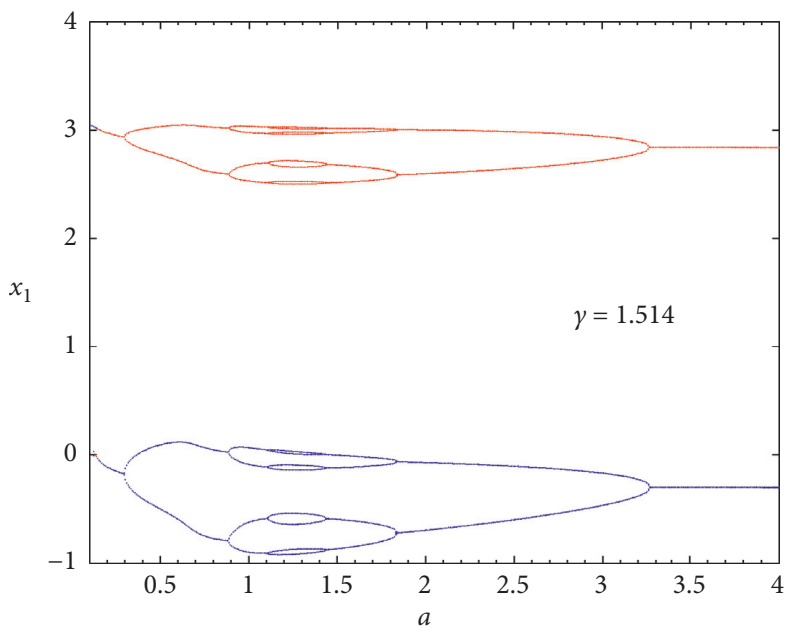

(c)

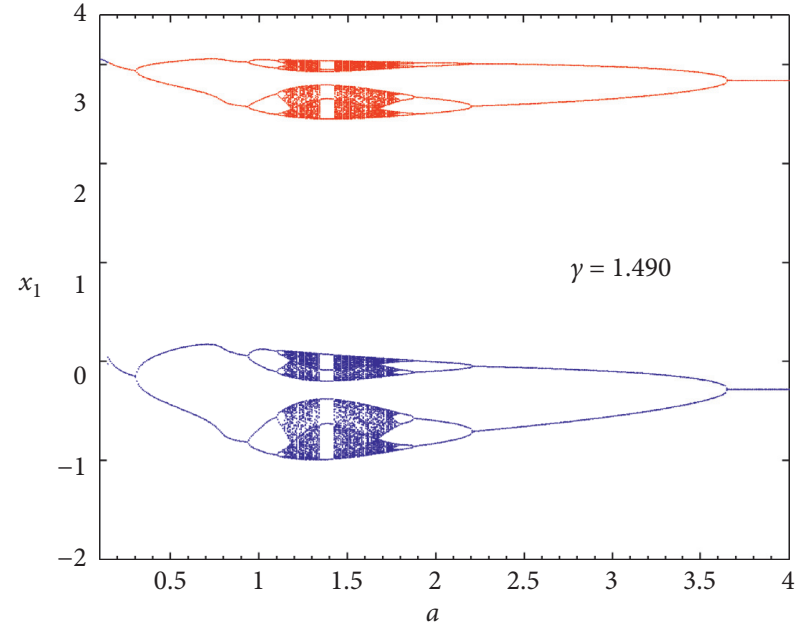

(b)

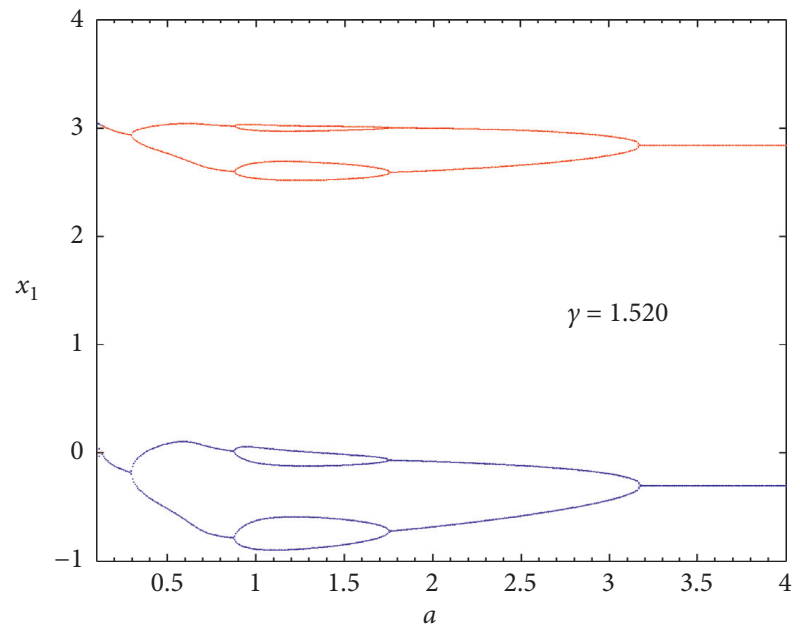

(d)

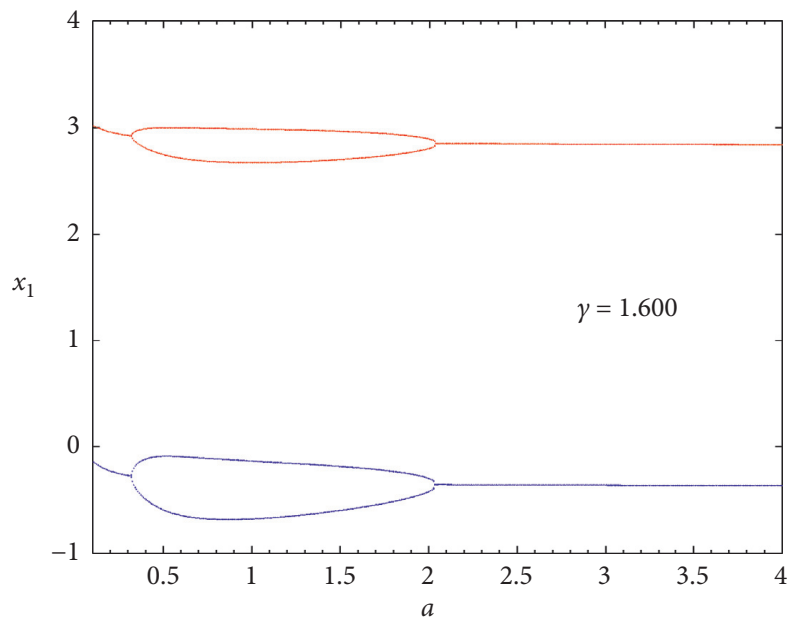

(e)

FIGURE 11: Bifurcation diagrams of the system showing local maxima of the coordinate $x_{1}$ versus the control parameter $a$ computed for some discrete values of $\gamma$ keeping $\mu=1.0$ and $k=0.00$. In each diagram, the blue and red branches are obtained by scanning the parameter downward (i.e., downward continuation) starting with initial conditions $(-0.5,0,0)$ and $(0.5,0,0)$, respectively. 


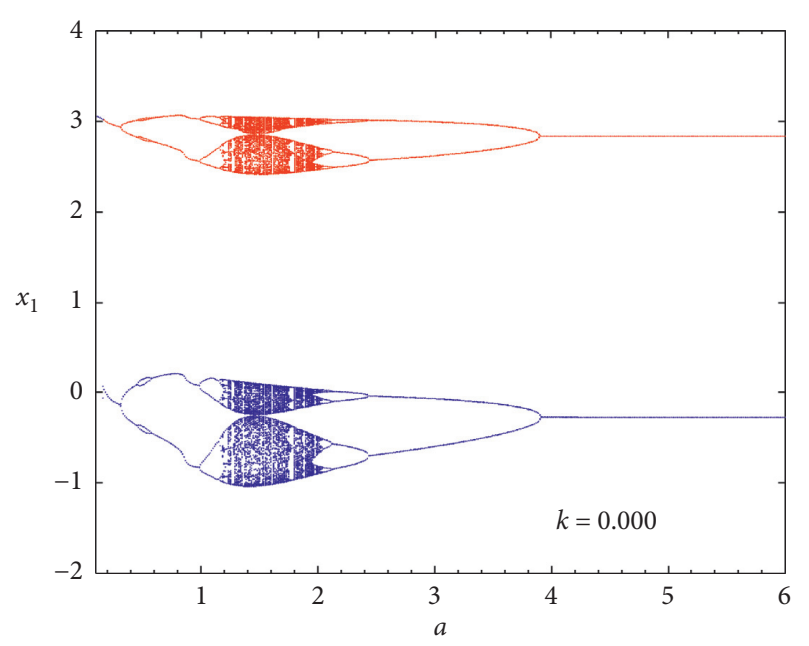

(a)

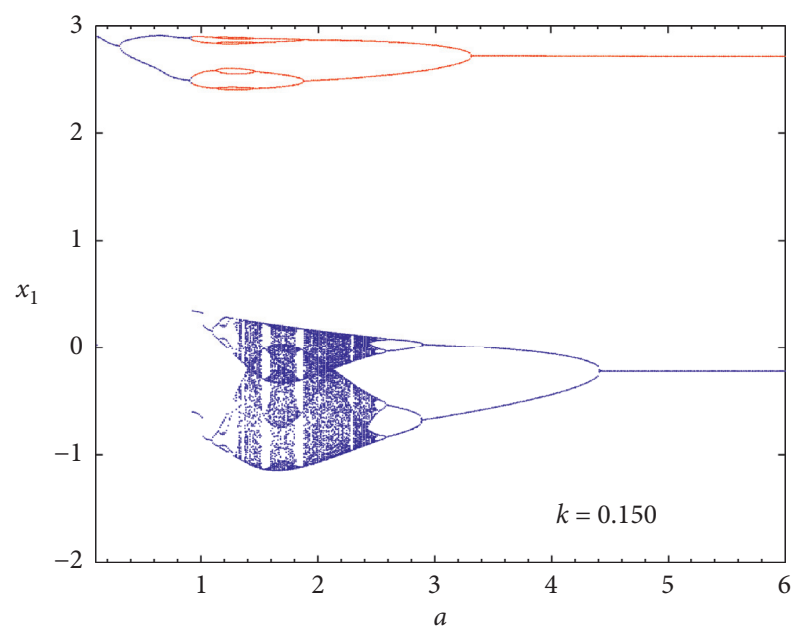

(c)

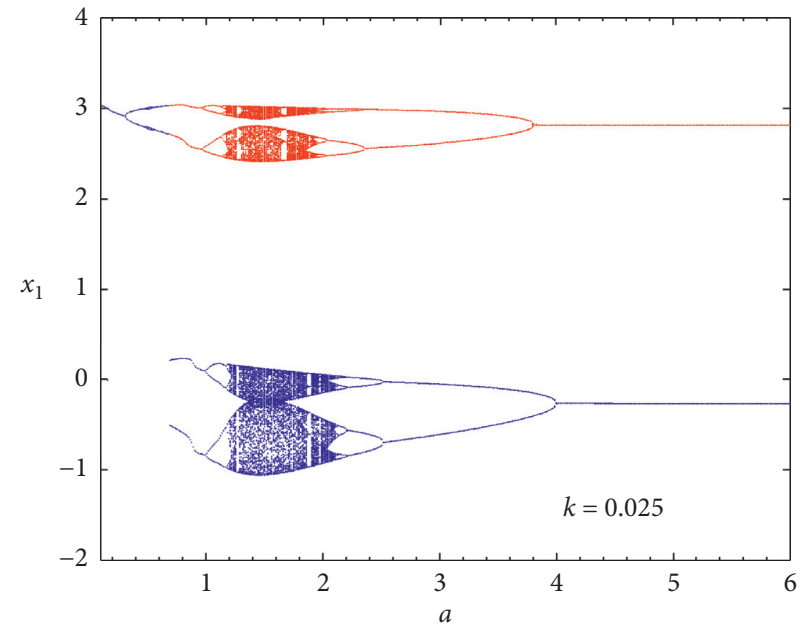

(b)

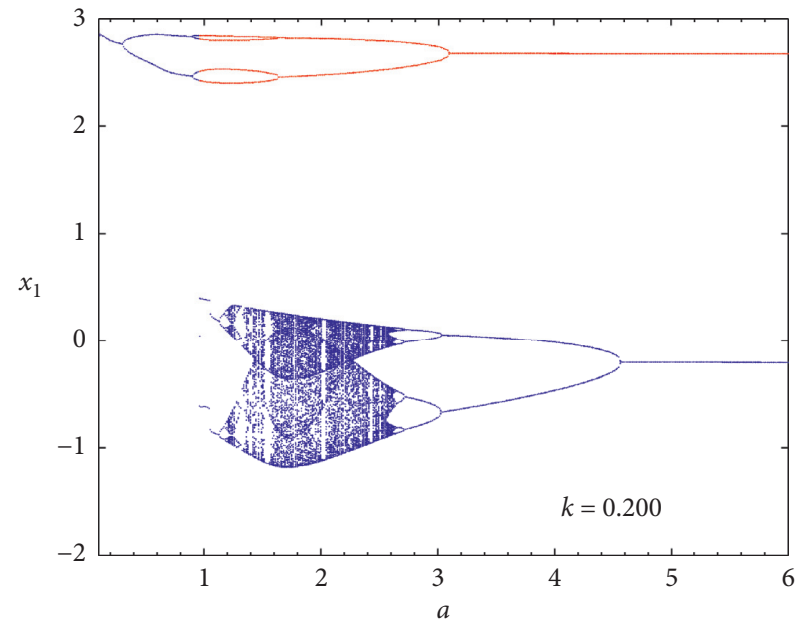

(d)

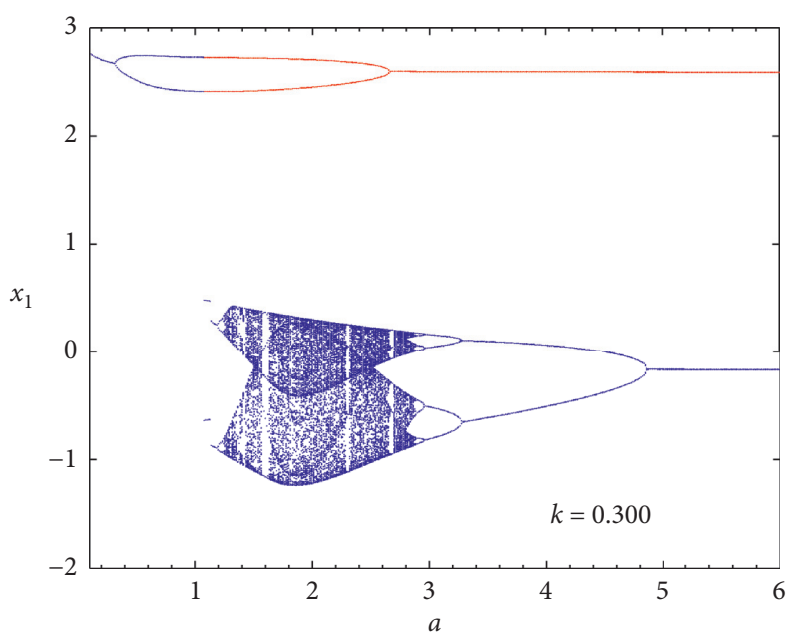

(e)

FIGURE 12: Bifurcation diagrams of the coordinate $x_{1}$ versus $a$ showing coexisting bubbles of bifurcation computed for five discrete value of $k$, keeping $\gamma=1.475$ and $\mu=1.0$. In each diagram, the blue and red branches are obtained by scanning the parameter downward (i.e., downward continuation) starting with initial conditions $(-0.5,0,0)$ and $(0.5,0,0)$, respectively. 


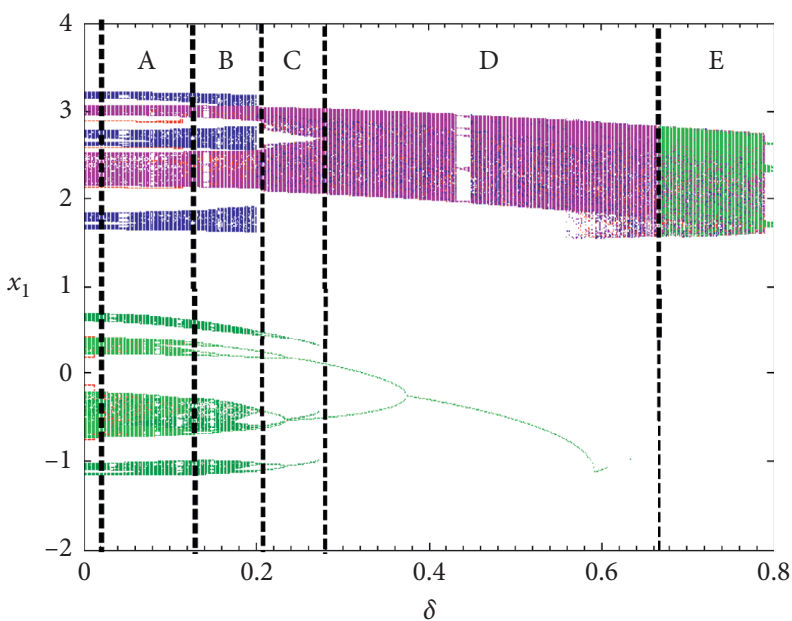

Figure 13: Bifurcation diagram illustrating the transition from a multistable state to monostability when smoothly varying the coupling strength in the range $0 \leq \delta \leq 0.80$. The rest of parameters are $a=17.77, \gamma=1.0$, and $\mu=0.95$. Regions $\mathrm{A}, \mathrm{B}, \mathrm{C}$, and $\mathrm{D}$ correspond to the coexistence of five, four, three, and two attractors, respectively, while a single attractor is observed in region E. Six sets of data are superimposed. These data are obtained by scanning the parameter upward starting from each of the six coexisting attractors (see text).

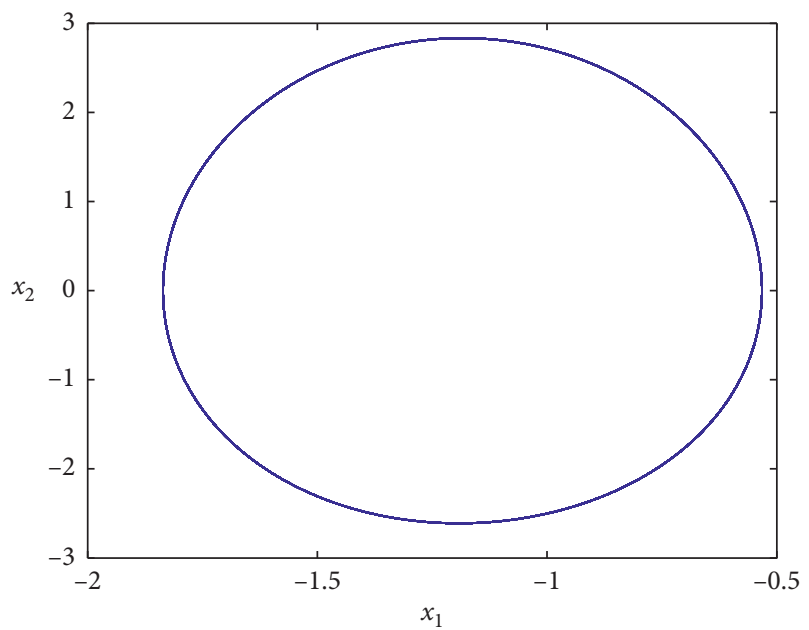

(a)

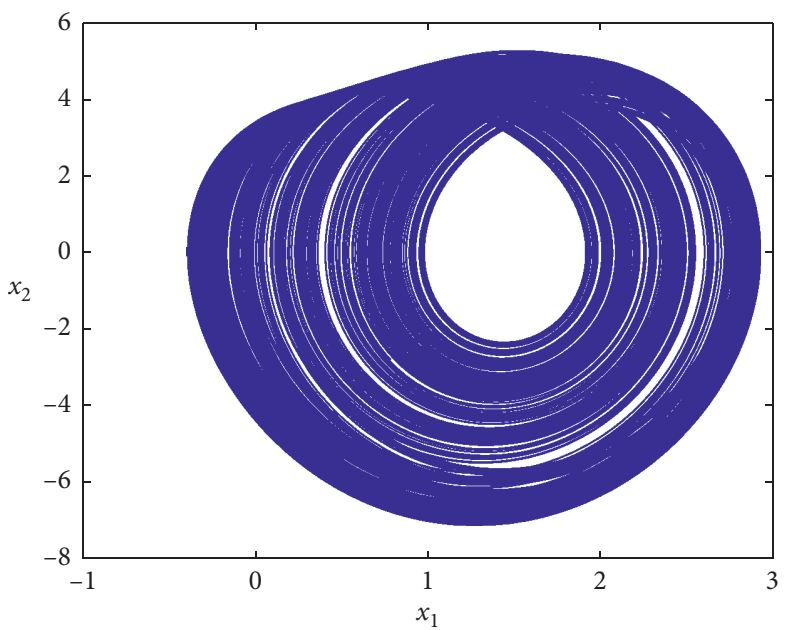

(b)

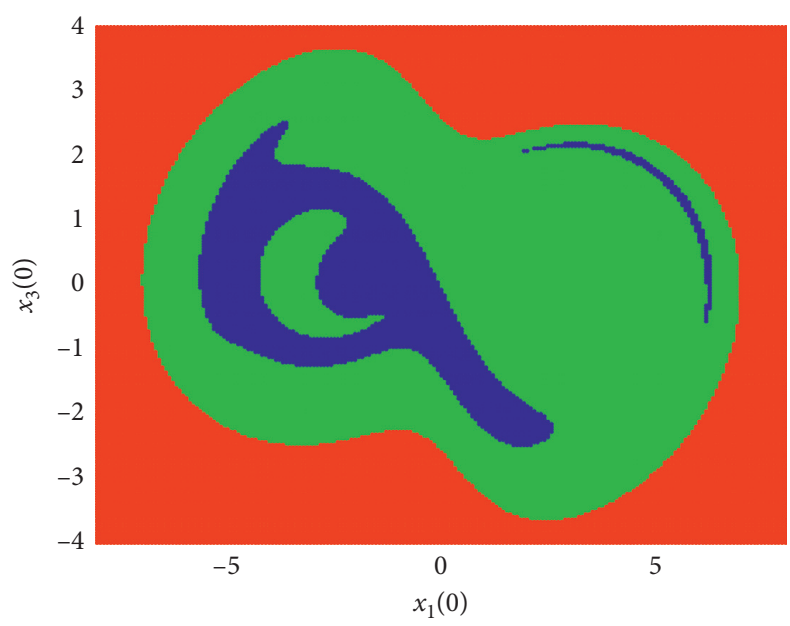

(c)

Figure 14: Coexistence of two different attractors for $\delta=0.50$ using two different initial conditions and corresponding cross section of the basins of attraction. Blue and green basins correspond, respectively, to the period-1 and the chaotic attractor, respectively, while red zone denotes unbounded dynamics. The rest of parameters are same as in Figure 18. 


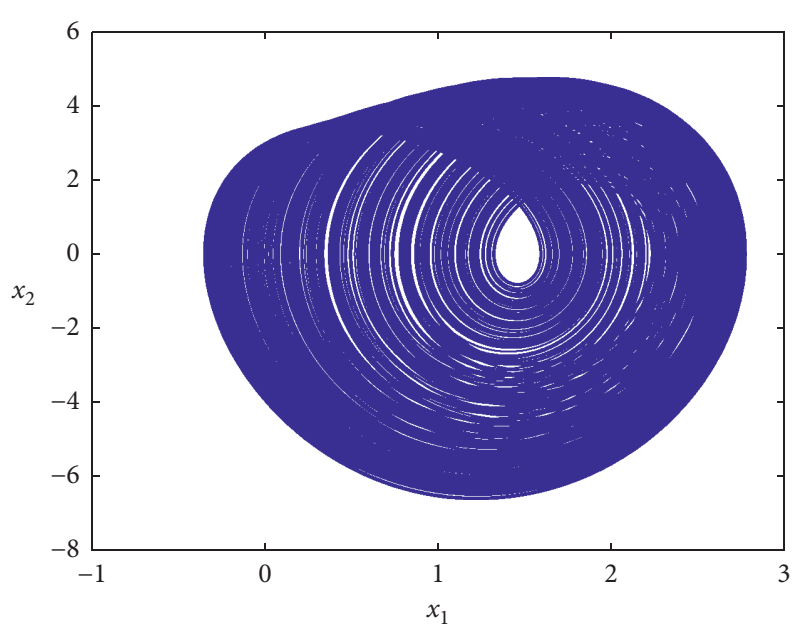

(a)

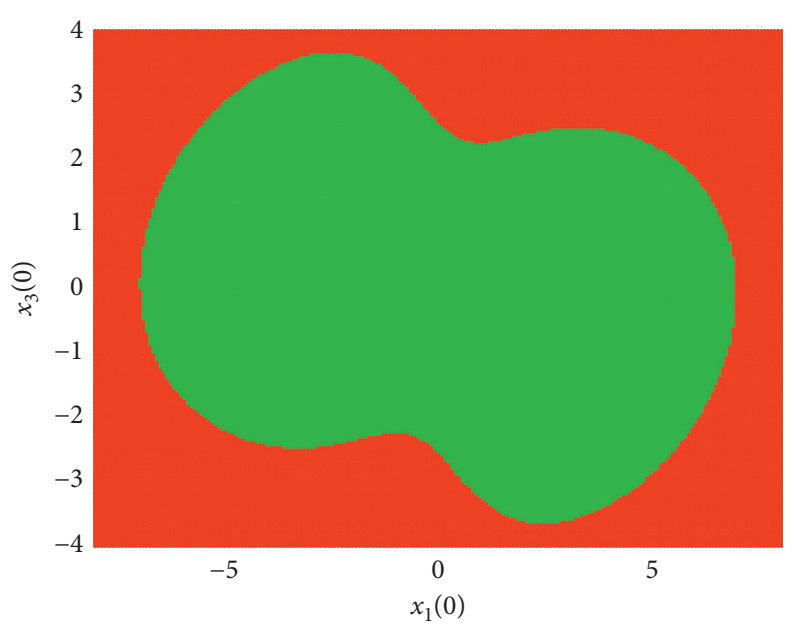

(b)

FIGURE 15: A single attractor (a) for $\delta=0.75$ and corresponding cross section of the basins of attraction (b). Green zones represent the basin of attraction of the chaotic attractor, while red zone denotes unbounded dynamics. The rest of parameters are same as in Figure 18.

coupled system. To this end, the parameters are fixed as in the caption of Figure 13. The latter figure shows the bifurcation diagrams illustrating the transition from a multistable state (see Figure 10) to monostability when smoothly varying the coupling strength in the range $0 \leq \delta \leq 0.80$. Regions $\mathrm{A}, \mathrm{B}, \mathrm{C}$, and $\mathrm{D}$ correspond to the coexistence of five, four, three, and two attractors, respectively, while a single attractor is observed in region E. Six sets of data are superimposed. These data are obtained by scanning the parameter upward starting from each of the six coexisting attractors without resetting the initial conditions. We present in Figures 15 and 16 sample phase portraits of the system (corresponding cross sections of the basins of attraction), highlighting the transition of the system to a monostable state.

\section{PSpice Simulations}

It is predicted from the above results that the jerk system with a single hyperbolic tangent function can undergo extremely varied dynamic behaviors. The design and implementation of a convenient electrical circuit (i.e., the analogue simulator) for the experimental study of the model are presented in this section. PSpice simulation [48] investigations are carried out to check the results of analytical and numerical analyses. The possibility of monitoring capacitors initial voltages and evaluating the corresponding impact on the behavior of the whole circuit represents one of the main advantages of using of PSpice. Interestingly, evidence of several coexisting stable solutions [49-54] in the system may easily be demonstrated both in the symmetric and the asymmetric modes of operation. Moreover, the hardware realization of theoretical chaotic mathematical models is convenient for engineering utilization including, for instance, random signal generation, chaos-based communications, and image encryption.
5.1. Design of the Experimental Circuit. The circuit diagram of the proposed electronic simulator is shown in Figures 16(a) and 16(b). The hyperbolic tangent nonlinearity module $[53,54]$ whose detailed schematic diagram is depicted in Figure 16(b) consists of resistors, a dualtransistor pair, a pair of operational amplifiers, and a dc current source. A detailed analysis of the hyperbolic tangent circuit can be found in [53, 54]. Operational amplifiers and related circuitry (in Figure 16(a)) implement the basic operations of addition, subtraction, and integration. By choosing a suitable time scaling, the simulator outputs can directly be displayed on the screen of a double trace oscilloscope by feeding the output voltage of $X_{1}$ to the $X$ input and the output voltage of $X_{2}$ to the $\mathrm{Y}$ input. With the hypothesis of ideal operational amplifiers operating in their linear regime, upon applying Kirchhoff current and voltage laws to the circuit diagram in Figure 16(a), it can be established that the voltages $X_{1}, X_{2}$, and $X_{3}$ satisfy the set of three coupled first-order nonlinear differential equations:

$$
\left\{\begin{array}{l}
\frac{d X_{1}}{d t_{e}}=\frac{X_{2}}{R C}, \\
\frac{d X_{2}}{d t_{e}}=\frac{X_{3}}{R_{a} C} \\
\frac{d X_{3}}{d t_{e}}=-\frac{V_{c c}}{R_{k} C}-\frac{3 X_{1}}{R C}-\frac{X_{2}}{R_{\gamma} C}-\frac{X_{3}}{R_{\mu} C}+\frac{6 \tanh \left(X_{1}\right)}{R C} .
\end{array}\right.
$$

Choosing the following rescale of time and variables: $t_{e}=$ $t R C ; X_{k}=x_{k} \times 1 V(k=1,2,3)$, system (10) is identical to system (1) with the following definition of parameters:

$$
a=R / R_{a} ; \quad \gamma=R / R_{\gamma} ; \quad \mu=R / R_{\mu} ; \quad k=\frac{R}{R_{k}} \frac{V_{c c}}{1 V} .
$$




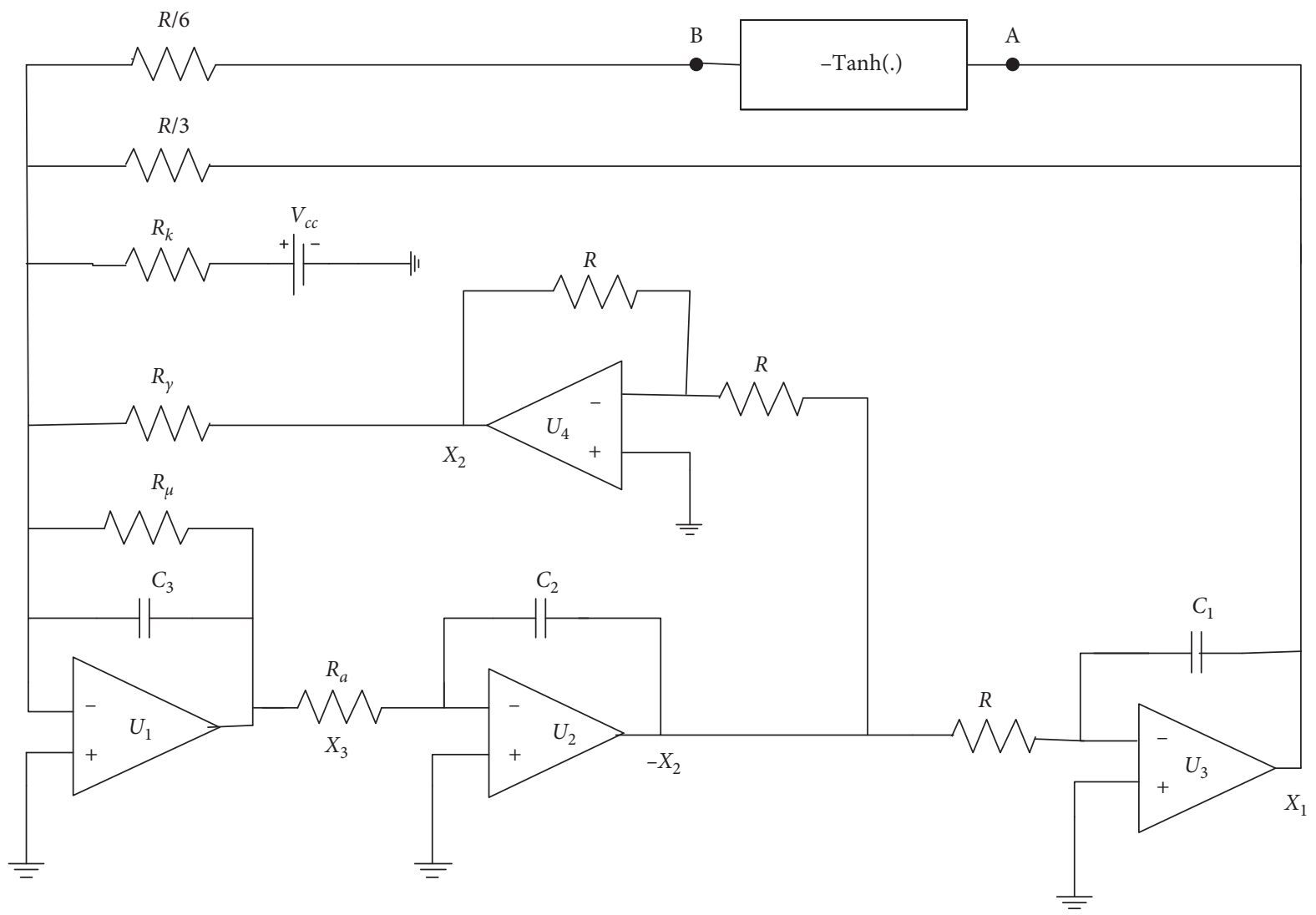

(a)

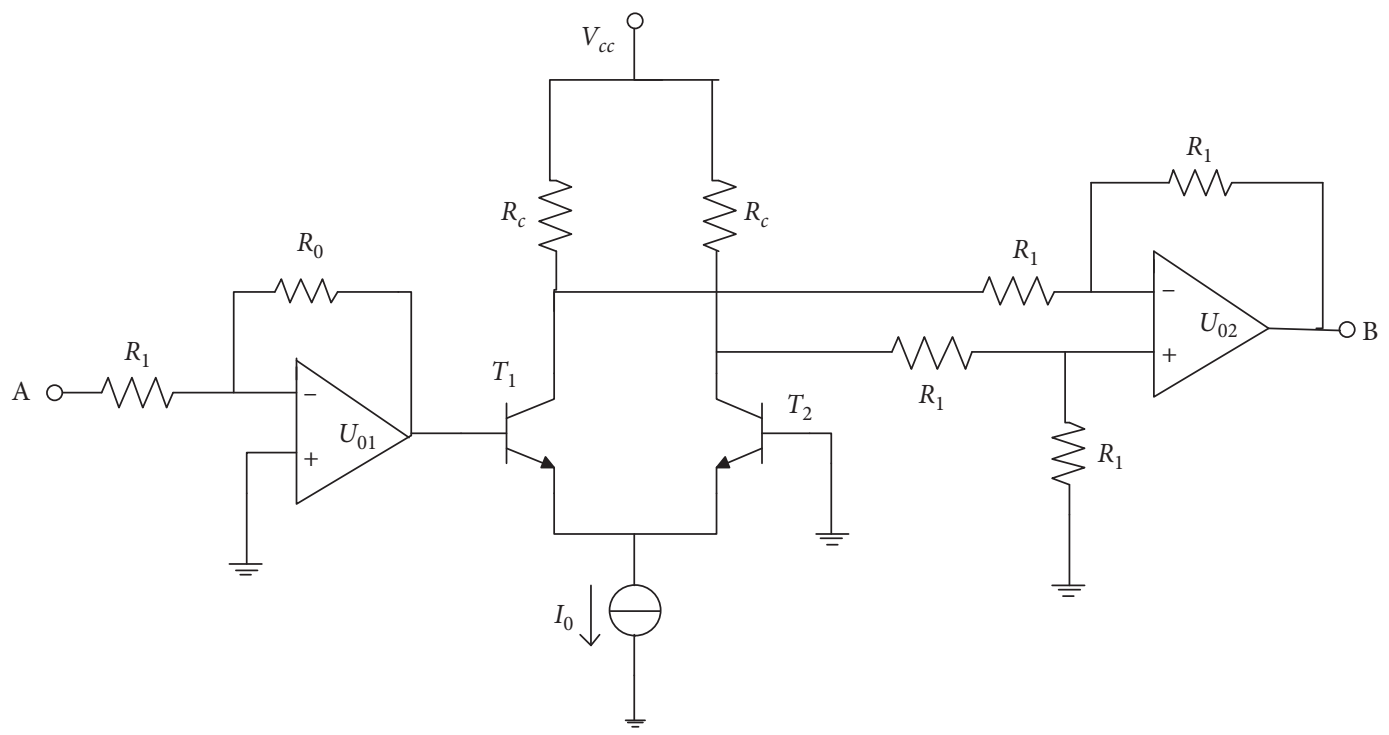

(b)

FIGURE 16: Electronic circuit implementation (a) of system. The circuit realization of tangent hyperbolic values function is shown in (b). The values of electronic circuit components used for the analysis are listed in Table 2.

From the above equations, it follows that the dynamics of system (1(a) and 1(b)) can be simulated at any desired frequency (within the bandwidth of op. amplifiers) by choosing the value of the three capacitors.
5.2. PSpice Simulation Results. The behavior of the circuit shown in Figure 16 is studied in PSpice by employing the values of parameters provided in Table 2 in order to check the theoretically predicted results of Section 3, in particular 
TABLE 2: The values of electronic components used for PSpice simulations.

\begin{tabular}{lcc}
\hline Parameters & Signification & Values \\
\hline$R_{0}$ & Resistance & $0.52 K \Omega$ \\
$R_{C}$ & Resistance & $1 K \Omega$ \\
$R_{1}$ & Resistance & $10 K \Omega$ \\
$R$ & Resistance & $12 K \Omega$ \\
$R_{a}$ & Tunable resistance & Tunable \\
$R_{\mu}$ & Resistance & Tunable \\
$R_{\gamma}$ & Resistance & Tunable \\
$C_{1}, C_{2}, C_{3}$ & Capacitance & $10 n F$ \\
$V_{C C}$ & Voltage source & $15 V_{D C}$ \\
$I_{0}$ & Current source & $1.1 m A$ \\
$T_{1}, T_{2}$ & Q & Q2N2222 \\
$U_{i}(i=1,2,3,4)$ & Amplifier transistors NPN & TL084 \\
$U_{01}, U_{02}$ & Operational amplifiers & TL082 \\
\hline
\end{tabular}

Figure 16 is partially reproduced from $(J)$ [18].

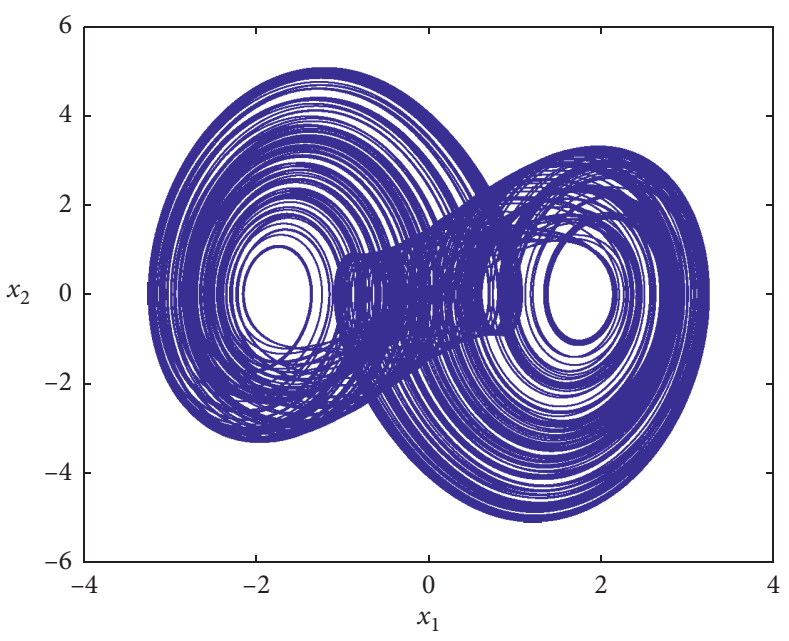

(a)

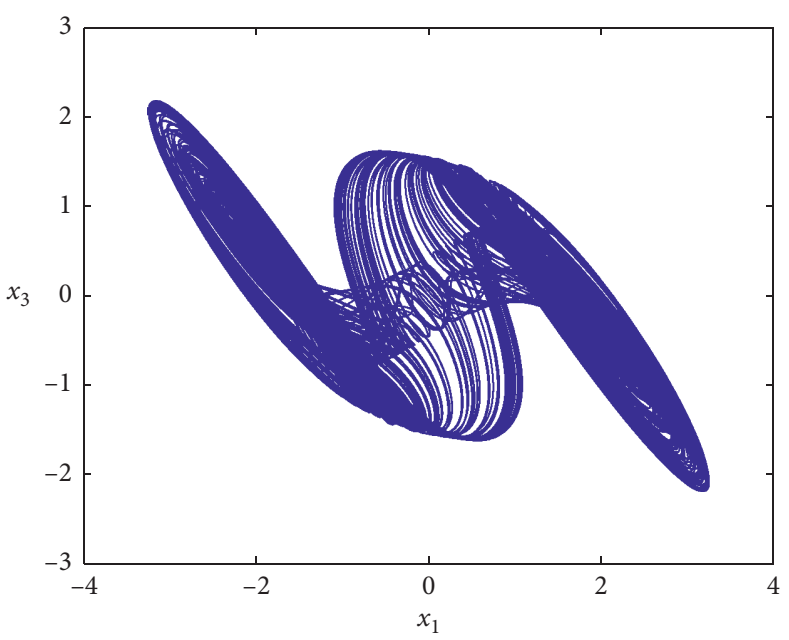

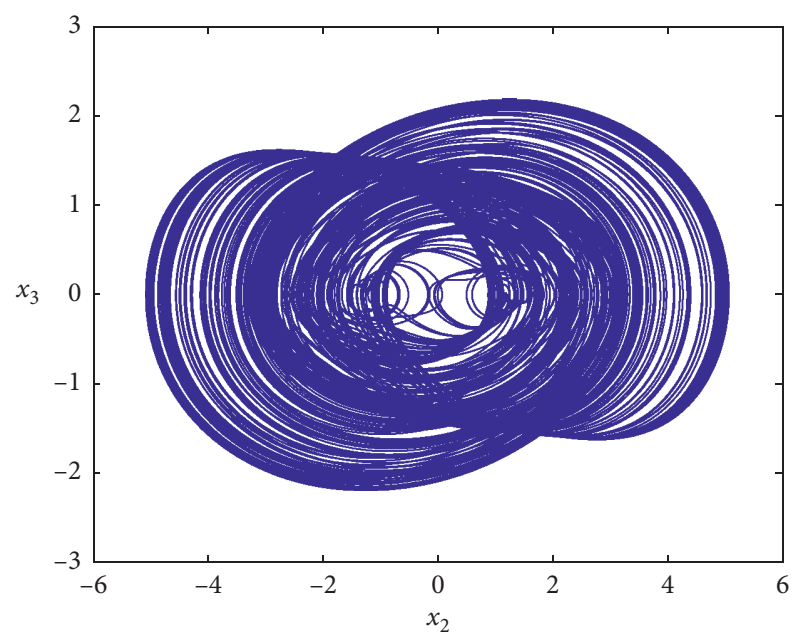

(b)

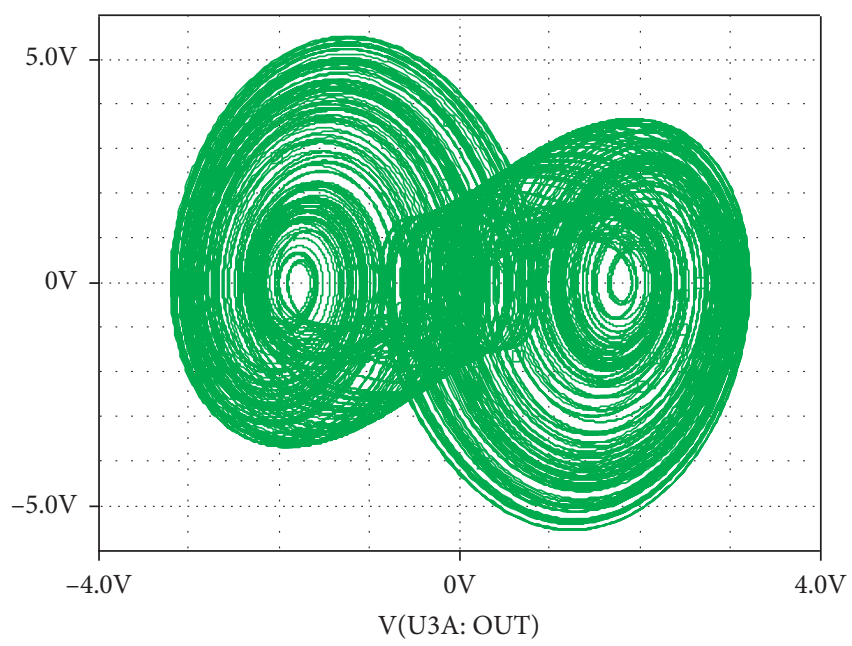

口 V(U4A: OUT)

(c)

(d)

Figure 17: Continued. 


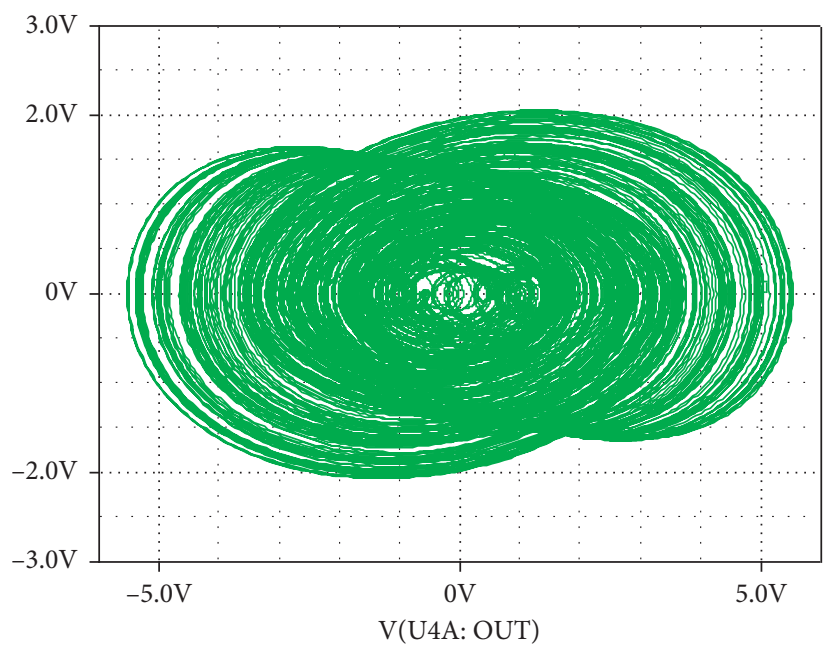

口 V(U1A: OUT)

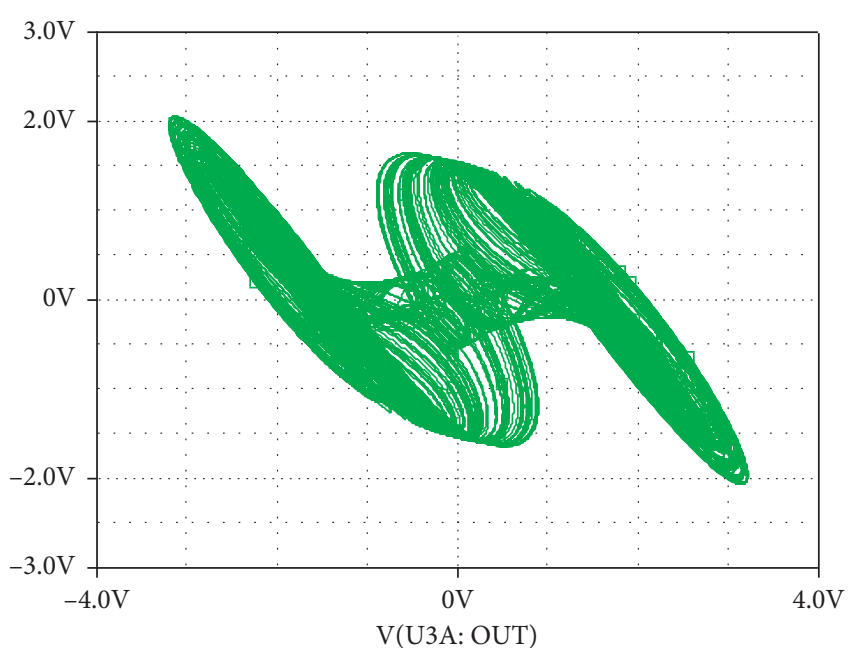

口 V(U1A: OUT)

(e)

(f)

Figure 17: Two-dimensional views (left panel) of the symmetric double-scroll chaotic attractors computed for $a=5.0, \mu=1.0$, and $k=$ 0.00 and the corresponding PSpice simulation results (right panel) obtained for $R_{\gamma}=11.13 \mathrm{k} \Omega$ and $R_{k}=\infty$ with the initial point $(0.5,0,0)$.

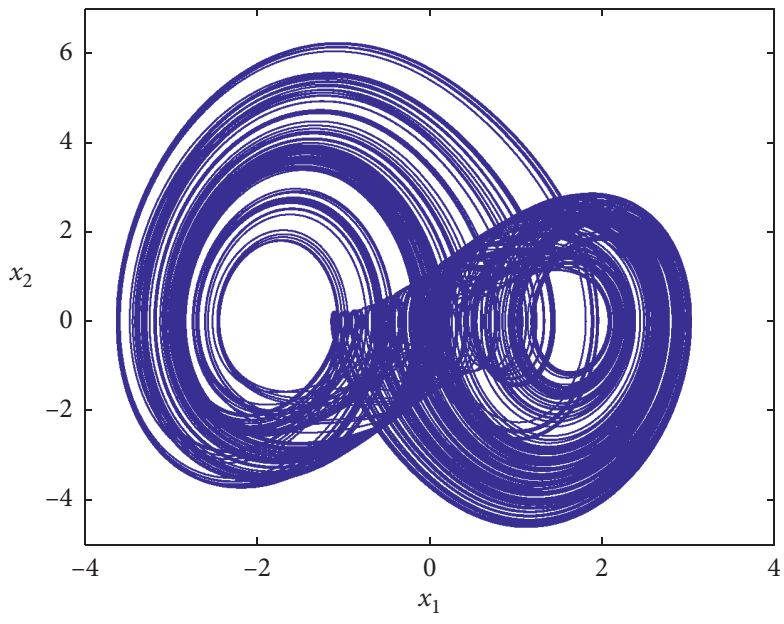

(a)

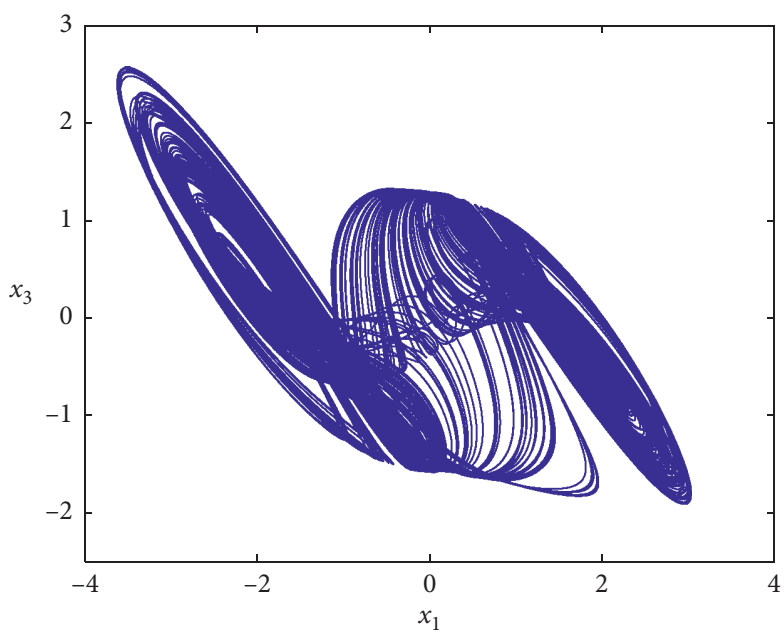

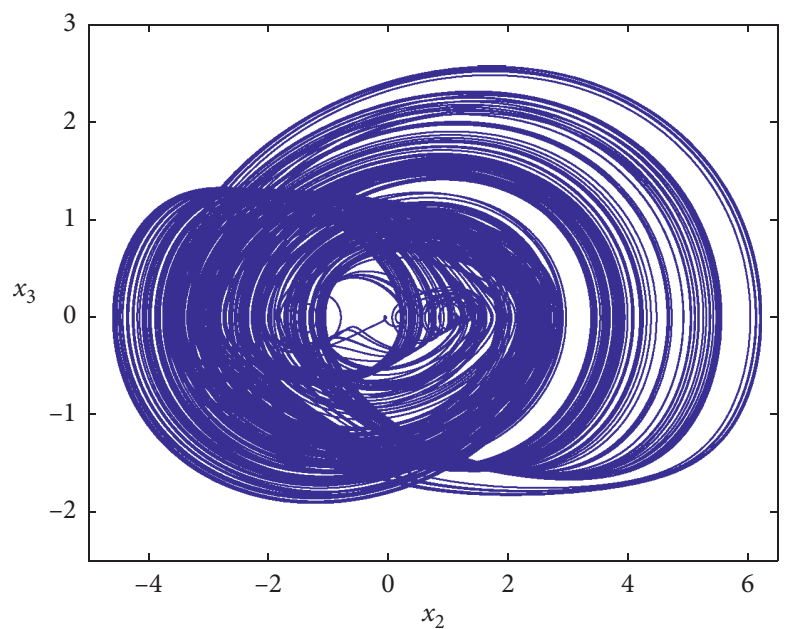

(b)

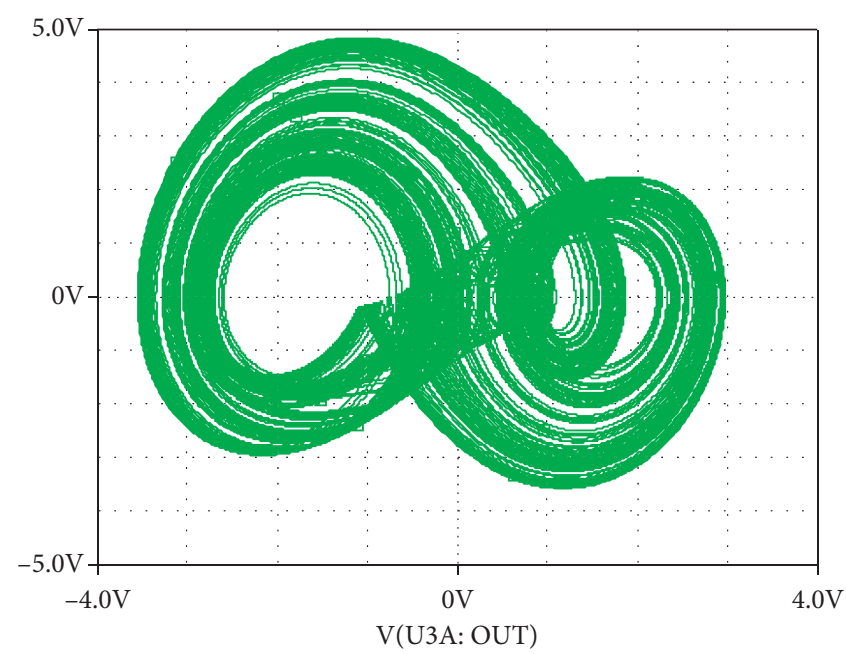

口 V(U1A: OUT)

(c) 


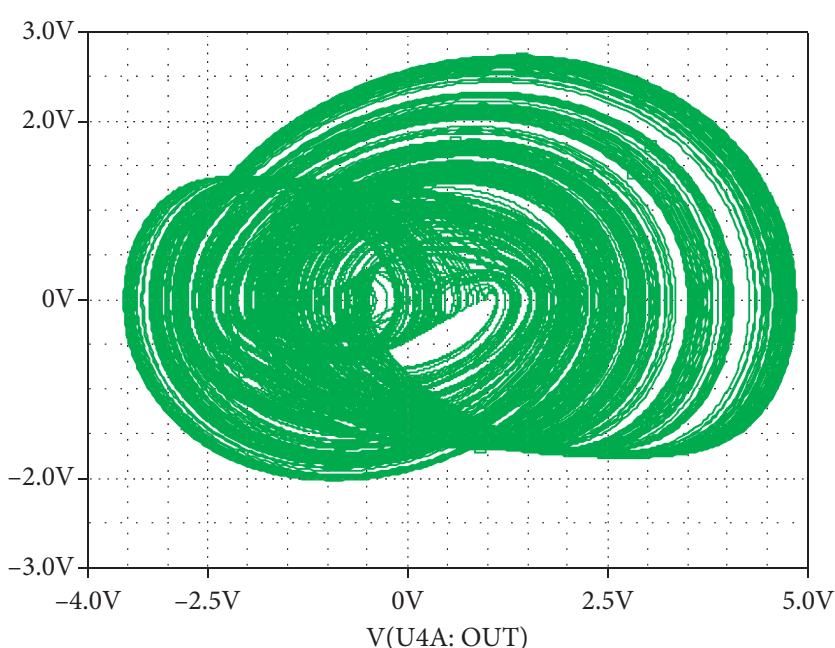

口 V(U1A: OUT)

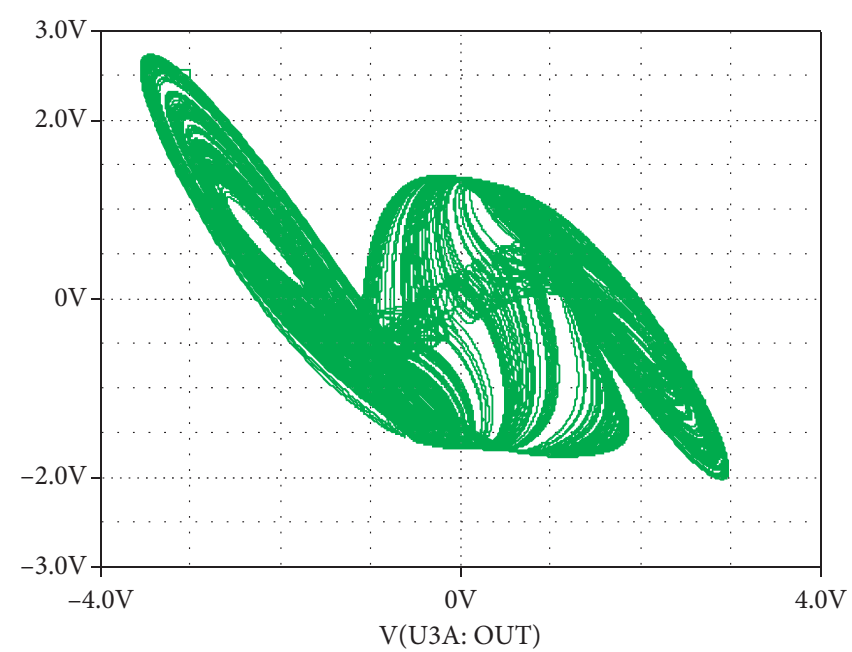

口 V(U1A: OUT)

(e)

(f)

FIgURe 18: Two-dimensional views (left panel) of the asymmetric double-scroll chaotic attractors computed for $a=5.0$ and $k=0.25$ and the corresponding PSpice simulation results (right panel) obtained for $R_{\gamma}=12 \mathrm{k} \Omega, R_{k}=576 \mathrm{k} \Omega$, and $R_{\mu}=12 \mathrm{k} \Omega$ with the initial point $(0.5,0,0)$.

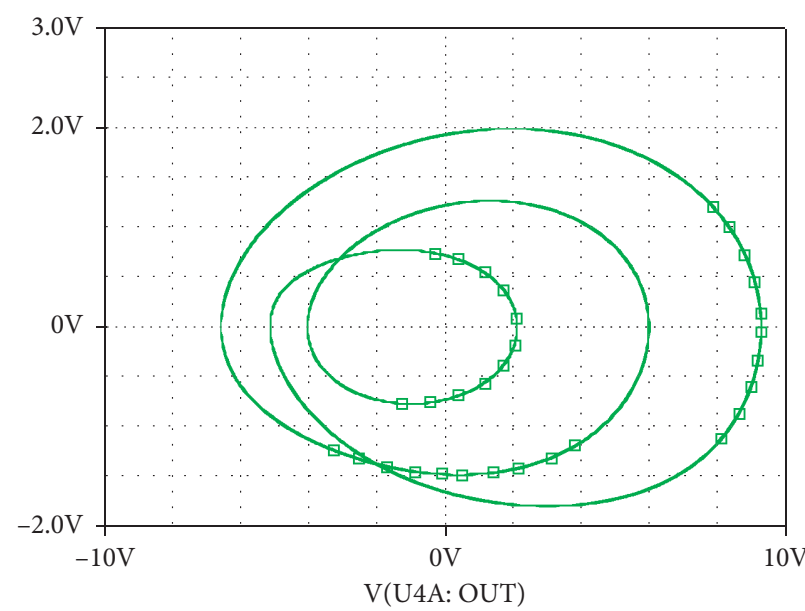

V(U1A: OUT)

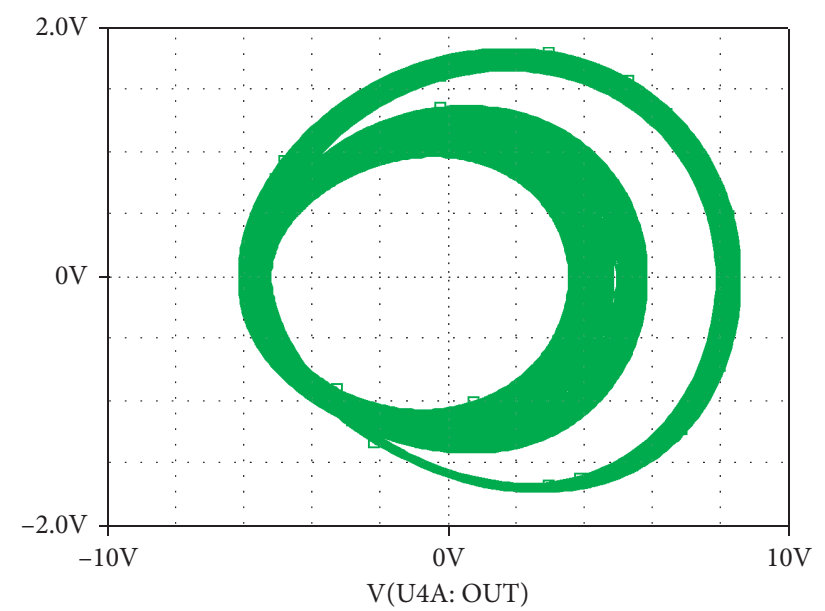

口 V(U1A: OUT)

(a)

(b)

FIgURE 19: Continued. 


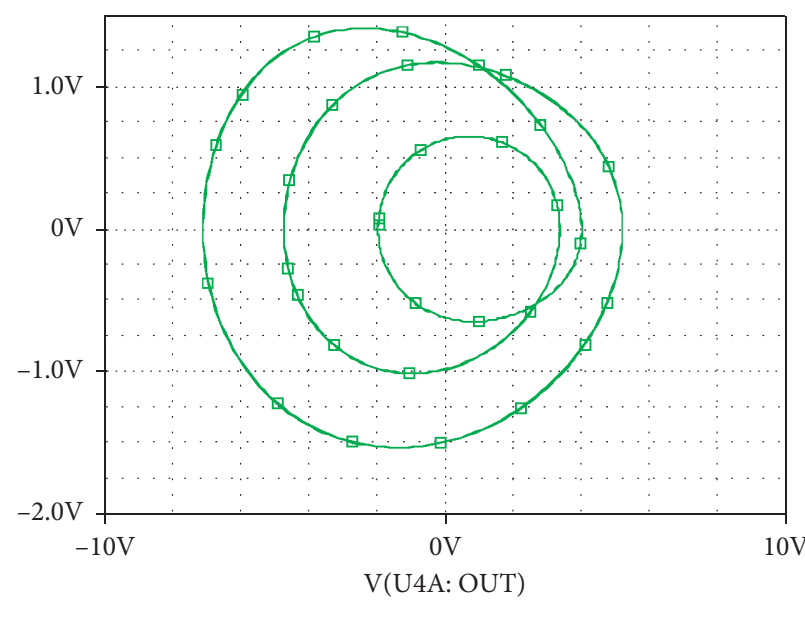

口 V(U1A: OUT)

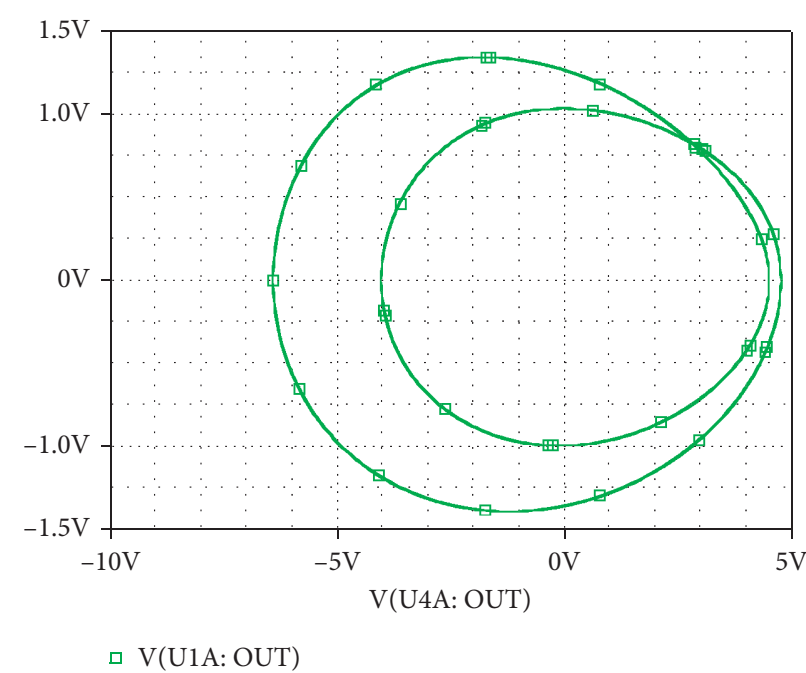

(d)

FIGURE 19: PSpice simulation results showing the coexistence of four different asymmetric attractors for $R_{a}=666 \Omega, R_{\mu}=12.631 \mathrm{k} \Omega$, $R_{\gamma}=12 \mathrm{k} \Omega$, and $R_{k}=576 \mathrm{k} \Omega$ obtained with four different initial conditions $\left(v_{c_{1}}(0), v_{c_{2}}(0), v_{c_{3}}(0)\right)$ : (a) a period-3 limit cycle for $(0.4,0.0,0.0)$; (b) a chaotic attractor for $(0.2,0.0,0.0)$; (c) a period-3 limit cycle for $(-0.303,0.0,0.0)$; (d) a period-2 limit cycle for $(-1.8,0.0,0.0)$.

the period doubling route to chaos and the presence of coexisting bifurcation branches. $R_{\gamma}$ (i.e., equivalently parameter $\gamma$ ) is chosen as main bifurcation control resistor. The electronic components' values $\left(R=12 \mathrm{k} \Omega, R_{\mu}=12 \mathrm{k} \Omega\right.$, $R_{a}=2.4 \mathrm{k} \Omega$, and $R_{k}=\infty$; see Table 2 ) are selected so as to match the dimensionless values $(a=5.0$ and $\mu=1.00)$ of Section 3 in view of allowing the comparison between theory and PSpice results. When varying progressive resistor $R_{\gamma}$, we observe the same sequence of bifurcations described in PSpice. Sample results showing the projections of the double-scroll attractor emanating from the merging crisis of coexisting asymmetric mono-scroll chaotic attractors for $R_{\gamma}=11.13 \mathrm{k} \Omega$ are provided in Figure 17 along with corresponding theoretical ones. Similarly, Figure 18 depicts various projections of the asymmetric double-scroll chaotic attractor obtained in PSpice (right panel) and the corresponding theoretically obtained ones (left panel) when the control resistors are fixed as $R_{\gamma}=12 \mathrm{k} \Omega, R_{k}=576 \mathrm{k} \Omega$, $R_{a}=2.4 \mathrm{k} \Omega$, and $R_{\mu}=12 \mathrm{k} \Omega$. The bifurcation sequences observed in PSpice perfectly agree with those of theoretical studies carried out in Section 3. On the contrary, using the electronic component values fixed as $R_{\gamma}=12 \mathrm{k} \Omega$, $R_{k}=576 \mathrm{k} \Omega, R_{a}=666 \Omega$, and $R_{\mu}=12.631 \mathrm{k} \Omega$, we have observed the coexistence of four different asymmetric attractors, namely, a pair of asymmetric period-3 cycles, a period-2 cycle, and a chaotic attractor when starting the system from four different initial conditions (see Figure 19). The latter situation is identical to the case reported in Figure 8 during the theoretical analysis. We have avoided the inclusion of other cases of multistability obtained in PSpice for the sake of brevity. We would like to point out the existence of some small shifts in the values of the control resistor $R_{\gamma}$ in PSpice in comparison to the theoretically predicted values. Such discrepancies are mainly due to the unavoidable simplifications adopted during the modeling step of the analogue simulator (e.g., ideal bipolar junction transistor model and ideal op. amplifier model, in comparison with more realistic/complex models implemented in PSpice).

\section{Concluding Remarks}

In summary, this paper has explored the dynamics of a simple chaotic jerk system with hyperbolic tangent nonlinearity whose symmetry is destroyed by the adding a constant term acting as an external excitation force. We have shown that the modified system exhibits several unusual and interesting nonlinear patterns such as coexisting bifurcation branches, hysteretic behaviors, coexisting symmetric and asymmetric bubbles, critical phenomena, and multiple (i.e., two, three, four, five, or six) coexisting asymmetric attractors for some appropriately chosen sets of its parameters. These features were illustrated by exploiting common nonlinear analysis tools such as graphs of largest Lyapunov exponent, bifurcation diagrams, phase portraits, and basins of attraction. The control of multistability based on the linear augmentation scheme is exploited to tune the system from the state of six coexisting attractors to monostability. An appropriate electronic analogue of the system was designed and simulated in PSpice. The theoretical results show a very good agreement with the PSpice simulation investigations.

The model considered in this work can be regarded as prototypal autonomous $3 \mathrm{D}$ system with three rest points and an odd symmetry. Also, we conjecture that the dynamics induced by symmetry break observed in this work may also be found when using the jerk equation with other types of nonlinearities (e.g., cubic, quintic, hyperbolic sine, and piece-wise quadratic). Moreover, the extension of the 
analysis presented in this paper to cases of other chaotic oscillators such as Chua's, Shinriki, autonomous van der Pol-Duffing, and hyperjerk circuits is under consideration.

\section{Data Availability}

The data used to support the findings of this study are available from the corresponding author upon request.

\section{Conflicts of Interest}

The authors declare that there are no conflicts of interest.

\section{References}

[1] S. H. Strogatz, Nonlinear Dynamics and Chaos Reading, Addison-Wesley, Boston, MA, USA, 1994.

[2] Y. A. Kuznetsov, Elements of Applied Bifurcation Theory, Springer-Verlag, New York, NY, USA, 1995.

[3] A. H. Nayfeh and B. Balachandran, Applied Nonlinear Dynamics: Analytical, Computational and Experimental Methods, John Wiley \& Sons, New York, NY, USA, 1995.

[4] J. C. Sprott, Elegant Chaos: Algebraically Simple Flow, World Scientific Publishing, Singapore, 2010.

[5] S. Jafari and J. C. Sprott, "Simple chaotic flows with a line equilibrium," Chaos, Solitons \& Fractals, vol. 57, pp. 79-84, 2013.

[6] S. Jafari, J. C. Sprott, and M. Molaie, "A simple chaotic flow with a plane of equilibria," International Journal of Bifurcation and Chaos, vol. 26, no. 6, p. 1650098, 2016.

[7] V. T. Pham, S. Jafari, C. Volos, A. Giakoumis, S. Vaidyanathan, and T. Kapitaniak, "A chaotic system with equilibria located on the rounded square loop and its circuit implementation," IEEE Transactions on Circuits and Systems II: Express Briefs, vol. 63, no. 9, pp. 878-882. In Press, 2016.

[8] V.-T. Pham, S. Jafari, C. Volos, S. Vaidyanathan, and T. Kapitaniak, "A chaotic system with infinite equilibria located on a piecewise linear curve," Optik, vol. 127, no. 20, pp. 9111-9117, 2016.

[9] V.-T. Pham, S. Jafari, J. Ma, X. Wang, and J. Ma, “A Chaotic system with different shapes of equilibria," International Journal of Bifurcation and Chaos, vol. 26, no. 4, p. 1650069 , 2016.

[10] A. N. Pisarchik and U. Feudel, "Control of multistability," Physics Reports, vol. 540, no. 4, pp. 167-218, 2014.

[11] C. Kahllert, "The effects of symmetry breaking in Chua's circuit and related piecewise-linear dynamical systems," Int. J. of Bif. and Chaos, vol. 3, no. 4, pp. 963-979, 1993.

[12] S. K. Dana, S. Chakraborty, and G. Ananthakrishna, "Homoclinic bifurcation in Chua's circuit," Pramana Journal of Physics, vol. 64, no. 3, p. 44344, 2005.

[13] A. Sofroniou and S. R. Bishop, "Breaking the symmetry of the parametrically excited pendulum," Chaos, Solitons \& Fractals, vol. 28, no. 3, pp. 673-681, 2006.

[14] S. R. Bishop, A. Sofroniou, and P. Shi, "Symmetry-breaking in the response of the parameterically excited pendulum model," Chaos Solitons and Fractals, vol. 25, no. 2, pp. 27-264, 2005.

[15] R. Rynio and A. Okniński, "Symmetry breaking and fractal dependence on initial conditions in dynamical systems: ordinary differential equations of thermal convection," Chaos, Solitons \& Fractals, vol. 9, no. 10, pp. 1723-1732, 1998.

[16] H. Cao and Z. Jing, "Chaotic dynamics of Josephson equation driven by constant dc and ac forcings," Chaos, Solitons \& Fractals, vol. 12, no. 10, pp. 1887-1895, 2001.
[17] M. Henrich, T. Dahms, V. Flunkert, S. W. Teitsworth, and E. Scholl, "Symmetry breaking transitions in networks of nonlinear circuits elements," New Journal of Physics, vol. 12, Article ID 113030, 2010.

[18] J. Kengne, S. M. Njikam, and V. R. F. Signing, "A plethora of coexisting strange attractors in a simple jerk system with hyperbolic tangent nonlinearity," Chaos, Solitons \& Fractals, vol. 106, pp. 201-213, 2018.

[19] J. C. Sprott, "A new chaotic jerk circuit," IEEE Transactions on Circuits and Systems II: Express Briefs, vol. 58, no. 4, pp. 240-243, 2011.

[20] P. Louodop, M. Kountchou, H. Fotsin, and S. Bowong, "Practical finite-time synchronization of jerk systems: theory and experiment," Nonlinear Dynamics, vol. 78, no. 1, pp. 597-607, 2014.

[21] J. Kengne and R. L. T. Mogue, "Dynamic analysis of a novel jerk system with composite tanh-cubic nonlinearity: chaos, multi-scroll, and multiple coexisting attractors," International Journal of Dynamics and Control, vol. 7, no. 1, pp. 112-133, 2018.

[22] J. Kengne, V. R. F. Signing, J. C. Chedjou, and G. D. Leutcho, "Nonlinear behavior of a novel chaotic jerk system: antimonotonicity, crises, and multiple coexisting attractors," International Journal of Dynamics and Control, vol. 6, no. 2, pp. 468-485, 2017.

[23] J. Kengne, Z. T. Njitacke, and H. B. Fotsin, "Dynamical analysis of a simple autonomous jerk system with multiple attractors," Nonlinear Dynamics, vol. 83, no. 1-2, pp. 751-765, 2016.

[24] S. Sedra and K. C. Smith, Microelectronic Circuits, Oxford University Press, London, UK, 2003.

[25] N. Özkurt, F. A. Savaci, and M. Gündüzalp, “The circuit implementation of a wavelet function approximator," Analog Integrated Circuits and Signal Processing, vol. 32, no. 2, pp. 171-175, 2002.

[26] D. Biswas and T. Banerjee, "A simple chaotic and hyperchaotic time-delay system: design and electronic circuit implementation," Nonlinear Dynamics, vol. 83, no. 4, pp. 2331-2347, 2016.

[27] W. Horton, R. S. Weigel, and J. C. Sprott, "Chaos and the limits of predictability for the solar-wind-driven magnetosphere-ionosphere system," Physics of Plasmas, vol. 8, no. 6, pp. 2946-2952, 2001.

[28] F. R. Tahir, R. S. Ali, V.-T. Pham, A. Buscarino, M. Frasca, and L. Fortuna, "A novel $4 \mathrm{D}$ autonomous $2 \$ \$ \backslash$ varvec $\{\mathrm{n}\} \$ \$ \mathrm{n}$ -butterfly wing chaotic attractor," Nonlinear Dynamics, vol. 85 , no. 4 , pp. 2665-2671, 2016.

[29] J. Kengne, Z. T. Njitacke, N. A Nguomkam, T. M Fouodji, and H. B. Fotsin, "Coexistence of multiple attractors and crisis route to chaos in a novel chaotic jerk circuit," International Journal of Bifurcation and Chaos, vol. 25, no. 4, Article ID 1550052, 2015.

[30] Z. T. Njitacke, J. Kengne, H. B. Fotsin, A. N. Negou, and D. Tchiotsop, "Coexistence of multiple attractors and crisis route to chaos in a novel memristive diode bidge-based Jerk circuit," Chaos, Solitons \& Fractals, vol. 91, pp. 180-197, 2016.

[31] V. Kirk and A. M. Rucklidge, "The effect of symmetry breaking on the dynamics near a structurally stable heteroclinic cycle between equilibria and a periodic orbit," $D y$ namical Systems, vol. 23, no. 1, pp. 43-74, 2008.

[32] J. Porter and E. Knobloch, "Dynamics in the 1:2 spatial resonance with broken reflection symmetry," Physica D: Nonlinear Phenomena, vol. 201, no. 3-4, pp. 318-344, 2005. 
[33] R. Lauterbach, "Symmetry breaking in dynamical systems," in Nonlinear Dynamical Systems and Chaos 1996; Progress in Nonlinear Differential Equations and Their Applications, H. W. Broer, S. A. van Gils, I. Hoveijn, and F. Takens, Eds., Vol. 19, Birkhäuser, Basel, Switzerland, 1996.

[34] C. Li and J. C. Sprott, "Amplitude control approach for chaotic signals," Nonlinear Dynamics, vol. 73, no. 3, pp. 1335-1341, 2013.

[35] C. Li, J. C. Sprott, A. Akgul, H. C. Lu, and Y. Zhao, "A new chaotic oscillator with free control," Chaos, vol. 27, Article ID 083101, 2017.

[36] G. A. Leonov and N. V. Kuznetsov, "Hidden attractors in dynamical systems. From hidden oscillations in HilbertKolmogorov, Aizerman, and Kalman problems to hidden chaotic attractor in Chua circuits," International Journal of Bifurcation and Chaos, vol. 23, no. 1, Article ID 1330002, 2013.

[37] G. A. Leonov, N. V. Kuznetsov, and T. N. Mokaev, "Hidden attractor and homoclinic orbit in Lorenz-like system describing convective fluid motion in rotating cavity," Communications in Nonlinear Science and Numerical Simulation, vol. 28, no. 1-3, pp. 166-174, 2015.

[38] A. Wolf, J. B. Swift, H. L. Swinney, and J. A. Vastano, "Determining Lyapunov exponents from a time series," Physica D: Nonlinear Phenomena, vol. 16, no. 3, pp. 285-317, 1985.

[39] S. P. Dawson, C. Grebogi, J. A. Yorke, I. Kan, and H. Koçak, "Antimonotonicity: inevitable reversals of period-doubling cascades," Physics Letters A, vol. 162, no. 3, pp. 249-254, 1992.

[40] U. Parlitz and W. Lauterborn, "Period-doubling cascades and devil's staircases of the driven van der Pol oscillator," Physical Review A, vol. 36, no. 3, pp. 1428-1434, 1987.

[41] L. Kocarev, K. S. Halle, K. Eckert, and L. O. Chua, "Experimental observation of antimonotonicity in chua's circuit," International Journal of Bifurcation and Chaos, vol. 3, no. 4, pp. 1051-1055, 1993.

[42] I. M. Kyprianidis, I. N. Stouboulos, P. Haralabidis, and T. Bountis, "Antimonotonicity and chaotic dynamics in a fourth-order autonomous nonlinear electric circuit," International Journal of Bifurcation and Chaos, vol. 10, no. 8, pp. 1903-1915, 2000.

[43] M. Bier and T. C. Bountis, "Remerging Feigenbaum trees in dynamical systems," Phys Lett A, vol. 104, pp. 239-244, 1994.

[44] B. Bao, L. Xu, N. Wang, H. Bao, Q. Xu, and M. Chen, "Thirdorder RLCM-four-elements-based chaotic circuit and its coexisting bubbles," AEU - International Journal of Electronics and Communications, vol. 94, pp. 26-35, 2018.

[45] P. R. Sharma, A. Singh, A. Prasad, and M. D. Shrimali, "Controlling dynamical behavior of drive-response system through linear augmentation," The European Physical Journal Special Topics, vol. 223, pp. 1531-1539, 2015.

[46] P. R. Sharma, M. D. Shrimali, A. Prasad, N. V. Kuznetsov, and G. A. Leonov, "Control of multistability in hidden attractors," The European Physical Journal Special Topics, vol. 224, no. 8, pp. 1485-1491, 2015.

[47] P. R. Sharma, M. D. Shrimali, A. Prasad, N. V. Kuznetsov, and G. A. Leonov, "Controlling dynamics of hidden attractors," International Journal of Bifurcation and Chaos, vol. 25, no. 4, pp. 1-7, 2015.

[48] D. C. Hamill, "Learning about chaotic circuits with SPICE," IEEE Transactions on Education, vol. 36, no. 1, pp. 28-35, 1993.

[49] Z.-H. Guan, Q. Lai, M. Chi, X.-M. Cheng, and F. Liu, "Analysis of a new three-dimensional system with multiple chaotic attractors," Nonlinear Dynamics, vol. 75, no. 1-2, pp. 331-343, 2014.
[50] Q. Lai and S. Chen, "Generating multiple chaotic attractors from sprott B system," International Journal of Bifurcation and Chaos, vol. 26, no. 11, p. 1650177, 2016.

[51] Q. Lai, A. Akgul, X.-W. Zhao, and H. Pei, "Various types of coexisting attractors in a new $4 \mathrm{D}$ autonomous chaotic system," International Journal of Bifurcation and Chaos, vol. 27, no. 9, p. 1750142, 2017.

[52] B. C. Bao, Q. D. Li, N. Wang, and Q. Xu, "Multistability in Chua's circuit with two stable node-foci," Chaos, vol. 26, no. 4, Article ID 043111, 2016.

[53] S. Duan and X. Liao, "An electronic implementation for Liao's chaotic delayed neuron model with non-monotonous activation function," Physics Letters A, vol. 369, no. 1-2, pp. 37-43, 2007.

[54] S. Duan and L. Wang, "A novel delayed chaotic neural model and its circuitry implementation," Computers \& Mathematics with Applications, vol. 57, no. 11-12, pp. 1736-1742, 2009. 